\title{
Experimental Study and Numerical Modeling of Fracture Propagation in Shale Rocks During Brazilian Disk Test
}

\author{
Mohaddeseh Mousavi Nezhad ${ }^{1} \cdot$ Quentin J. Fisher $^{2} \cdot$ Elia Gironacci $^{1} \cdot$ Mohammad Rezania $^{1}$ (D)
}

Received: 24 February 2017 / Accepted: 30 January 2018 / Published online: 3 March 2018

(c) The Author(s) 2018. This article is an open access publication

\begin{abstract}
Reliable prediction of fracture process in shale-gas rocks remains one of the most significant challenges for establishing sustained economic oil and gas production. This paper presents a modeling framework for simulation of crack propagation in heterogeneous shale rocks. The framework is on the basis of a variational approach, consistent with Griffith's theory. The modeling framework is used to reproduce the fracture propagation process in shale rock samples under standard Brazilian disk test conditions. Data collected from the experiments are employed to determine the testing specimens' tensile strength and fracture toughness. To incorporate the effects of shale formation heterogeneity in the simulation of crack paths, fracture properties of the specimens are defined as spatially random fields. A computational strategy on the basis of stochastic finite element theory is developed that allows to incorporate the effects of heterogeneity of shale rocks on the fracture evolution. A parametric study has been carried out to better understand how anisotropy and heterogeneity of the mechanical properties affect both direction of cracks and rock strength.
\end{abstract}

Keywords Fracking $\cdot$ Crack propagation $\cdot$ Heterogeneous shale rock $\cdot$ Stochastic modeling

\section{Introduction}

Hydraulic fracturing, commonly known as fracking, is an important process in extracting gas from shale formations. Accurate predictions of the directions and the extent of fractures are required in order to conduct safe and economically viable operations for gas production. Fractures tend to propagate along the direction of the least resistance path. The direction and extent of this path is a complex function of the in situ stress condition, anisotropic mechanical properties of the rock and pore and fracture fluid pressures (Warpinski and Smith 1989). It is possible to evaluate the influence of the in situ stress condition and pore-pressure variations on the evolution of fracture networks. This could be done using data from relevant laboratory tests, deep borehole logs from the field and coupled fluid-solid computational codes. However, the role of spatial variability and anisotropy of

Mohammad Rezania

M.Rezania@warwick.ac.uk

1 School of Engineering, The University of Warwick, Coventry CV4 7AL, UK

2 School of Earth and Environment, The University of Leeds, Leeds LS2 9JT, UK mechanical properties on the crack propagation remains poorly understood.

Numerous experimental and numerical studies, using a variety of techniques, have been performed for understanding the behavior of different types of rocks (Wong and Einstein 2009; Morgan et al. 2013). Less effort has been placed on the characterization of the mechanical properties of shale rocks. Study of the effect of bedding direction on crack propagation is a topic of great interest; recently, Morgan and Einstein (2014) conducted an experimental investigation on the effects of bedding laminations on crack propagation in the Opalinus shale samples and concluded that the shear strength and elastic modulus of shale samples as well as fracture advancement strongly depend on bedding laminates. The experimental observations showed that the direction of stress-induced cracks which are generally perpendicular to the maximum principal stress changes toward the bedding orientations. These tests are crucial to understand the effect of material composition and local heterogeneities on the mechanical response of rocks, which, because of their natural genesis, significantly differ from one to another (Bobko and Ulm 2008; Veytskin et al. 2017). Hou et al. (2016) compared the behavior of shale, coal and sandstone specimens subjected to high-pressure fluid-driven 
fracturing, and observed how different microscopic composition of these materials leads to important differences in the measured values of mechanical properties, strains and crack patterns. Kim et al. (2012) also performed a comparative study between shale, gneiss and schist rocks samples, investigating the influence of their composition and direction of bedding planes on their thermo-mechanical properties.

The proper determination of mechanical properties of fracturing rock plays a fundamental role in predicting the mechanical response of the rock. Fracture toughness, the resistance of the rock to crack propagation, is considered to be a significant factor in determining the artificially created fracture network patterns. In particular, there is an extreme scarcity of published data and knowledge on the fracture toughness and elastic modulus of sedimentary geomaterials such as shales. It is particularly crucial to gain reasonable understanding of the fundamental processes controlling fracture propagation in shale rocks. This necessitates the undertaking of experimental and numerical studies to correctly assess the strength of these materials and identify the geomechanical characterization of these heterogeneous rocks.

Tests conducted on Brazilian disks specimens which are traditionally used to indirectly calculate rock's tensile strength prove significant influence of laminations inclination on the mechanical response of the materials (Wang et al. 2016; Mokhtari and Tutuncu 2016; Mousavi Nezhad et al. 2018). Zhong et al. (2015) also studied the effect of damage at microscale on the overall macroscopic response of the shale samples subjected to triaxial compression and highlighted that both bedding inclinations and different confining pressures influence on the compressive strength, elastic modulus, crack morphology and opening modes. Holt et al. (2015) also studied the effect of local heterogeneities on the material response and rock brittleness. Mahanta et al. (2017) investigated the importance of understanding the effect of the applied load on shale specimens and how, for varying strain rates, the fracture toughness, and consequently the energy required for fracturing the material, changes.

Duan and Kwok (2015) and Zhou et al. (2016) modeled the Brazilian disk problem and a fluid-driven fracture problem, respectively, in shale using discrete element method (DEM). However, the main issue with DEM model is the computational cost, which becomes prohibitive if largescale problems need to be analyzed. Classical finite element method (FEM) (Dokhani et al. 2016; Zeng and Wei 2017) and extended FEM (Sun et al. 2016) have been successfully applied to fracture propagation and fracking simulations on shale rocks. Anisotropy has been included in some of these numerical simulations by decreasing the values of mechanical properties, such as elastic modulus, cohesion coefficients or fracture toughness, in precise locations of the domain in order to create weak layers which affect fracture extents and shapes. However, Chen et al. (2016) showed that the introduction of weak bands is not enough to entirely reproduce the real anisotropic mechanical response of the shale rocks. It should be considered that in addition to the presence of the voids and microfractures, there are many types of inclusions, such as quartz, calcite and dolomite, forming a locally heterogeneous structure for the shales. In order to capture the real behavior of such heterogeneous materials, an indepth knowledge of their microstructure and comprehensive numerical implementations are required (Chen et al. 2016; Mousavi Nezhad et al. 2016).

In the context of numerical modeling, strategies for characterizing the random heterogeneity in materials can be categorized as either multi-scale homogenization or spatially varying random fields. The former is attractive from the point of view that the different phases in a material such as the matrix, inclusions and the interfaces are modeled through identifying a representative volume element and computing the effective properties of complex microstructures (Belytschko et al. 2008). However, the applicability of the method is limited to the cases that a clear separation of scales exists and a closed-form macroscopic equation can be driven to describe the relevant microstructural details, e.g., morphology or constituent material properties (Geers et al. 2010; Novák et al. 2012). Spatially varying random field-based approaches are able to directly approximate the randomness through generation of spatial realizations of the properties associated with a given correlation structures, and the crack trajectory is modeled using FEM within the context of Monte Carlo simulation (Yang and Xu 2008; Stefanou 2009).

An attempt to use probability theory for modeling layered materials has been proposed by Bossi et al. (2016), in which the authors modeled a simplified slope made up by clay with horizontal gravel layers. A probabilistic technique has been used to assign the same deterministic value of gravel's cohesion and friction angle to randomly selected elements forming the domain. This approach improved the reliability of numerical results, but mechanical properties have been deterministically defined. Borghi et al. (2015) also used a stochastic approach for introducing fractures with random dimensions and locations in the model. A noteworthy approach has been recently outlined by $\mathrm{Li}$ et al. (2016a, b) where material heterogeneity has been considered through a combined stochastic methodology accounting for different types of natural materials on a soil profile. Although this approach appears to be very promising, it does not account for material microstructures, which is well known to considerably influence the macroscopic behavior (Bobko and Ulm 2008; Gironacci et al. 2017).

This paper focuses on geomechanical characterization of shales and investigates the anisotropic mechanical behavior of shale specimens under Brazilian test conditions, as well 
as the effects of random orientation of shale layers on the failure mechanisms. The paper also reports original laboratory measurements on the mechanical properties of core samples of shale-gas reservoir rocks collected from the Dove's Nest site on the east coast of North Yorkshire, in England. The data are presented on the general mechanical behavior of these rocks to delineate the basic parameters that control the propagation of cracks in shale-gas rocks. Elastic modulus and fracture toughness of shale specimens with different microstructural formations and layering orientations are calculated using Brazilian tests. A new computational modeling framework for simulation of crack propagation in heterogeneous layered materials is developed. It combines random field and continuum damage theories, and Weibull distribution and its spatial autocorrelation length information are used for generating samples of stochastic fields representing the layering structure as well as local heterogeneity of the shales. The numerical data are also discussed to better understand how anisotropy of the properties affects the morphology and direction of cracks.

\section{Fracture Advancement Methodology}

\subsection{The Variational Approach}

The basis of the variational approach to fracture mechanics relies on the association of a potential energy consisting of stored elastic energy, the work of external forces and the energy released through fracture to any crack and deformation configuration. A reference configuration $\subset \mathbb{R}^{N}, N=2$, of a homogeneous elastic body is considered which contains a generic crack as visualized in Fig. 1 .

The total energy of the body is defined as

$\mathcal{E}(f, K)=\int_{\Omega \backslash K} W(F(x)) \mathrm{d} x+\gamma \mathcal{H}^{N-1}(K)$

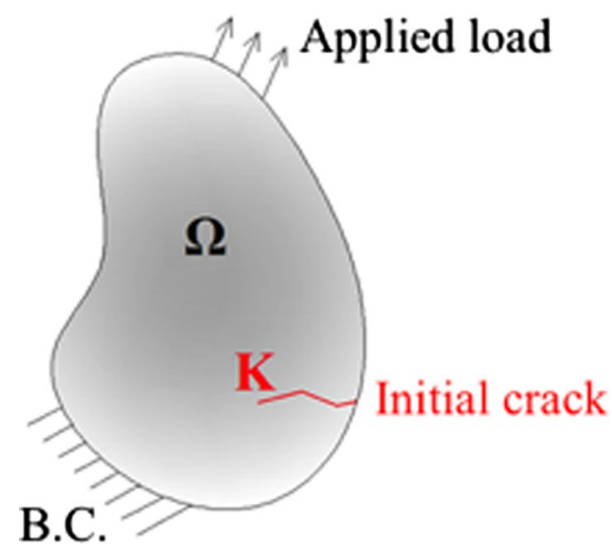

Fig. 1 Schematic configuration of a body with initial crack and applied boundary conditions where $f$ is the body deformation, $K \subset \bar{\Omega}$ is the fractured zone, $W: \mathbb{R}^{N x N} \rightarrow \mathbb{R}$ is the stored energy function of a hyperelastic material, $F$ is the deformation gradient, $\gamma$ is the fracture energy, and $\mathcal{H}^{N-1}$ is the Hausdorff measure of $K$ which provides the measure of the length of the crack for sufficiently regular fractured zone. The first and the second terms on the right hand side of Eq. (1) represent bulk and surface energy of the body, respectively.

Equation (1) can be minimized, so crack growth is deduced by successive minimization of energy at fixed time steps. The minimization of Eq. (1) with respect to any kinematically admissible displacement and any set of crack curves introduces a high level of complexity for the variation calculus of free-discontinuity problems, particularly due to the presence of non-smooth values of the $K$ parameter. In fact, unless topological constrains are added, it is not usually possible to deduce compactness properties from the only information that such kind of energies are bounded. Following the methodology proposed by De Giorgi and Ambrosio (1988), we introduce $K$ as a set of discontinuity points $S_{f}$ of the function $f$ and set the problems in a space of discontinuous functions. The weak formulation of the energy, by replacing the term $K$ with a set of discontinuity points $S_{f}$ of deformation in a Sobolev space $\operatorname{SBV}\left(\Omega ; \mathbb{R}^{N}\right)$, is therefore given by

$\mathcal{E}(f)=\int_{\Omega \backslash K} W(\nabla f) \mathrm{d} x+\gamma \mathcal{H}^{N-1}\left(S_{f}\right)$.

The presence of the term $\mathcal{H}^{N-1}\left(S_{f}\right)$ creates challenges in terms of finite element discretization of the functional. To overcome such challenges, Eq. (2) has been approximated, in the sense of $\Gamma$-convergence (Bourdin et al. 2000), by a family of numerically more tractable functionals defined over a Generalized Sobolev space GSBV. GSBV consists of all functions whose truncations are in Sobolev space $\left(\Omega ; \mathbb{R}^{N}\right)$ and allows extending the definition of Eq. (2) to $L^{1}$ functions, which are not of bounded variation. $\Gamma$-convergence makes it possible to achieve a variational convergence. This means if a minimizer $\vartheta_{\varepsilon}$ for a function $\mathcal{F}_{\varepsilon}$ exists for every $\varepsilon>$ 0 and if there is a sequence $h \mapsto \varepsilon_{h}$ such that $\varepsilon_{h} \rightarrow 0$ and the corresponding $\vartheta_{\varepsilon_{h}}$ converges to $\vartheta$, then $\vartheta$ is a minimizer for $\mathcal{F}_{\varepsilon}$. Based on the regularized formulation of the energy function for brittle fracture problems presented by Bourdin et al. (2000), an auxiliary variable $s$, which is called the damage parameter, is introduced. $s$ is a regularized representation of the fractured zone defining the discontinuity set in Eq. (2). Therefore, a functional space $\boldsymbol{X}$ can be considered which its elements are pairs $(f, s)$. Then, we take the functional $X \rightarrow$ $[0,+\infty]$ defined by

$\mathcal{F}(\boldsymbol{f}, \boldsymbol{s})= \begin{cases}\mathcal{E}(\boldsymbol{f}) & \text { if } f \in \mathcal{D}, s \equiv 1 \\ +\infty & \text { otherwise }\end{cases}$ 
with $\mathcal{E}(\boldsymbol{f})$ as in the problem, $\mathcal{D}$ is the domain of the functions $\in$ GSBV, the problem $\left(\mathcal{P}_{0}\right)$ defined as $\min \{\mathcal{F}(\boldsymbol{f}, \boldsymbol{s}):(\boldsymbol{f}, \boldsymbol{s}) \in \boldsymbol{X}\}$ is considered. Damage parameter provides a picture of the damage state of the body; for an undamaged and intact body, $\boldsymbol{s}$ is equal to 1 everywhere, while it goes to zero in proximity of the discontinuity set $S_{f}$.

The functional formulation for a generic $p>1$ is expressed in the form provided by Ambrosio and Tortorelli (1992)

$$
\begin{aligned}
\mathcal{F}_{\varepsilon}(f, s)= & \int_{\Omega}\left(s^{2}(x)+\kappa_{\varepsilon}\right) W(F(x)) \mathrm{d} x \\
& +\gamma \int_{\Omega^{\prime}}\left(\frac{\varepsilon^{p-1}}{p}|\nabla s(x)|^{p}+\frac{c}{\varepsilon p^{\prime}}(1-s(x))^{p}\right) \mathrm{d} x
\end{aligned}
$$

where $p$ is the $p$ th power of the norm of the function defined in the Sobolev space, $p^{\prime}=p /(p-1)$, $c=\left(2 \int_{0}^{1}(1-t)^{p / p^{\prime}} \mathrm{d} t\right)^{-p^{\prime}}$ is the normalization constant, $\kappa_{\varepsilon}$ is a positive regularization parameter, and $\varepsilon$ is related to the material length scale. Bulk and surface terms are two integrations over two different domains $\Omega$ and $\Omega^{\prime}$, physical domain and logical domain, respectively. $\Omega^{\prime}$ is defined as open set such that

$\Omega \subset \Omega^{\prime}, \quad \partial_{2} \Omega \subset \partial \Omega^{\prime}, \quad$ int $\partial_{1} \Omega \cap \partial \Omega^{\prime}=\not$

where $\partial_{1} \Omega$ and $\partial_{2} \Omega$ are the two disjoint parts of the boundary of $\Omega$, and $\operatorname{int} \partial_{1} \Omega$ is the interior of $\partial_{1} \Omega$ relative to $\partial \Omega$. The choice of the size of the logical domain is made on the consideration that it has to be big enough to avoid underestimation of the fracture energy when the crack reaches the boundary $\partial_{1} \Omega$.

For two-dimensional problem where $p=2$, the total energy formulation for the body can be represented as

$$
\begin{aligned}
\mathcal{F}_{\varepsilon}(f, s)= & \int_{\Omega_{0}}\left(s^{2}(x)+\kappa_{\varepsilon}\right) W\left(\nabla_{0} f\left(x_{0}\right)\right) \mathrm{d} x_{0} \\
& +\frac{\gamma}{2} \int_{\Omega_{0}^{\prime}}\left(\varepsilon\left|\nabla_{0} s\left(x_{0}\right)\right|^{2}+\frac{1}{\varepsilon}\left(1-s\left(x_{0}\right)\right)^{2}\right) \mathrm{d} x_{0}
\end{aligned}
$$

where $\Omega_{0}$ and $\Omega_{0}^{\prime}$ represent initial unfractured and stress-free configuration of the body in the physical and logical domain, respectively.

\subsection{Stored Energy Formulation}

Following Del Piero et al. (2007), we used an isotropic, compressible neo-Hookean type stored energy model that is defined as

$W(\boldsymbol{F})=\frac{\mu}{2}(\operatorname{tr} \boldsymbol{C}-2)+\Psi(J)$

where $\mu$ is the Lamé's second parameter, and $\boldsymbol{C}$ is the right Cauchy-Green tensor. In the above equation, the first term represents the classical formulation of an incompressible neo-Hookean material and the second term is a convex function that is defined as (Del Piero et al. 2007)

$\Psi(J)=\left\{\begin{array}{ll}\frac{\lambda}{2}(\ln J)^{2}-\mu \ln J & 0 \leq J \leq \dot{j} \\ \frac{\lambda}{2}(\ln J)^{2}-\mu \ln J+(\lambda \ln J-\mu)\left(\frac{J-j}{J}\right) & J \geq \dot{j}\end{array}\right.$,

where $\mu$ is the Lamé's first parameter and $j=\mathrm{e}^{(\lambda+\mu) / \lambda}$. Equation (7) is directly related to surface deformation, as it is function of the Jacobian of the deformation gradient $(J)$. As $J$ goes to zero the stored energy function goes to infinity, penalizing the extreme compression. To account for the tension-compression asymmetry of damage behavior of material, the methodology proposed in Li et al. $(2016 \mathrm{a}, \mathrm{b})$ is followed, and the energy function is decomposed into two parts; a positive part which is considered to contribute to damage, and a negative part that resists to damage:

$W(\boldsymbol{F})=W^{+}+W^{-}$

where

$W^{+}=\left.\left(s^{2}+k_{\varepsilon}\right) W\right|_{J>1}$

$W^{-}=\left.\left(1+k_{\varepsilon}\right) W\right|_{J<1}$

In the above equations, it can be noticed that the damage parameter appears only in the positive part of the energy function, the part associated with the elements that increase in surface (i.e., in the elements with $J>1$ ), the value for damage in the elements is kept as calculated. The elements that decrease in surface (i.e., the elements with $J<1$ ) do not contribute in damage. In this way, different behaviors for tension and compression are explicitly taken into account.

\subsection{Numerical Solution Strategy}

An approximation solution of the minimization of Eq. (5) is achieved using an iterative procedure, shown in algorithm (5), which consists of imposing a stationary condition to one of the deformation and damage variables, while keeping the other variable fixed. For all $v \in W^{1, d}\left(\Omega, \mathbb{R}^{n}\right), w \in W^{l, d}\left(\Omega_{0}\right)$, we look for a deformation that satisfies the stationary condition of

$$
\begin{aligned}
\delta \mathcal{F}_{\varepsilon}\left(\boldsymbol{f}_{n}, s_{n-1}\right)[v, 0]= & \int_{\Omega_{0}}\left(s_{n-1}^{2}\left(x_{0}\right)+k_{\mathcal{E}}\right) S\left(\nabla_{0} \boldsymbol{f}_{n}\left(x_{0}\right)\right) . \\
& \nabla_{0} v\left(x_{0}\right) \mathrm{d} x_{0}=0,
\end{aligned}
$$

and then for the scalar field $s$ stationary condition of

$$
\begin{aligned}
\delta \mathcal{F}_{\varepsilon}\left(f_{n}, s_{n}\right)[0, w]= & \int_{\Omega_{0}} 2 W\left(\nabla_{0} \boldsymbol{f}_{n}\right) s_{n} w \mathrm{~d} x_{0}+G \int_{\Omega_{0}^{\prime}} \varepsilon \nabla_{0} s_{n} . \\
& \nabla_{0} w-\frac{\left(1-s_{n}\right) w}{\varepsilon} \mathrm{d} x_{0} .
\end{aligned}
$$


where $\boldsymbol{S}(\boldsymbol{F})=\frac{\partial}{\partial \boldsymbol{F}} W(\boldsymbol{F})$ is the first Piola-Kirchhoff stress tensor.

By taking the updated Lagrangian formulation of Eq. (11) and its linearization (Del Piero et al. 2007) the following is obtained

\subsection{Stochastic Approach}

In order to incorporate the effects of material heterogeneity in the computational model, a statistical technique is employed. The Weibull distribution function (Weibull 1939)

$\int_{\Omega_{n-1}}\left(s_{n-1}^{2}+k_{\varepsilon}\right)\left((\operatorname{det} \boldsymbol{F})^{-1}(\boldsymbol{I} \bigotimes \boldsymbol{F})[\boldsymbol{S}(\boldsymbol{F})]+(\operatorname{det} \boldsymbol{F})^{-1}(\boldsymbol{I} \bigotimes \boldsymbol{F}) \frac{\partial S(\boldsymbol{F})}{\partial F}(\boldsymbol{I} \bigotimes \boldsymbol{F})^{T}\left[\nabla \bar{u}_{n}\right]\right) \cdot \nabla v \mathrm{~d} x=0$

where using Eq. (7)

$\mathbf{S}(\boldsymbol{F})=\left\{\begin{array}{l}\mu \boldsymbol{F}+(\lambda \ln J-\mu) \boldsymbol{F}^{-T} \quad 0 \leq J \leq \dot{z} \\ \mu \boldsymbol{F}+\lambda \mathrm{e}^{-(\lambda+\mu) / \lambda} J \boldsymbol{F}^{-T} \quad J \geq \dot{j}\end{array}\right.$

Applying the integration by parts, the final weak form of Eq. (12) is derived as

$$
\int_{\Omega_{0}} 2 W\left(\nabla_{0} f_{n}\right) s_{n} w \mathrm{~d} x_{0}-G \int_{\Omega_{0}^{\prime}}\left(\varepsilon \Delta_{0} s_{n}-\frac{1-s_{n}}{\varepsilon}\right) w \mathrm{~d} x_{0}=0
$$

MATLAB Partial Differential Equation Toolbox together with the Newton-Raphson iteration scheme is used to solve the above equations. The iteration stops when two consecutive pairs of solution $\left(f_{n-1}, s_{n-1}\right)$ and $\left(f_{n}, s_{n}\right)$ are close enough according to an identified convergence criterion. In order to avoid the healing of the cracks, an approximation method was used to consider irreversibility condition for damage evolution. We followed the methodology proposed by Del Piero et al. (2007). Based on that, irreversibility condition of $s_{n}(x)=s_{n-1}(x)$ if $s_{n}(x)>s_{n-1}(x)$ is set, and the value of damage parameter associated with each point in the body cannot exceed the one calculated at the previous time step. We leave the development of more advanced and rigorous methods for incorporating the irreversibility condition to future works. is used to generate random distributions of the properties, as it has a simple structure and its applicability for modeling failure of brittle materials has been verified (Fang and Harrison 2002; Yang and Xu 2008; Gorjan and Ambrožič 2012). In this study, the fracture energy is considered as a random variable.

Cumulative density function (CDF) and probability density function (PDF) for Weibull distribution, plotted in Fig. 2, take the form of

$P(\xi)=1-\exp \left(-\left(\frac{\xi}{\xi_{0}}\right)^{m}\right)$

$p(\xi)=\frac{\mathrm{d} P(\xi)}{\xi}=\frac{m}{\xi_{0}}\left(\frac{\xi}{\xi_{0}}\right)^{m-1} \exp \left(-\left(\frac{\xi}{\xi_{0}}\right)^{m}\right)$

where $\xi$ is the random parameter, $m$ is the shape parameter, and $\xi_{0}$ is the scale parameter.

In order to define both shape and scale parameters, maximum likelihood estimator (MLE) is used. Let $\xi_{1}, \xi_{2}, \ldots \xi_{n}$ be a random sample of size $n$ with a PDF $f_{\xi_{i}}\left(\xi_{i}, m, \xi_{0}\right)$, where $\xi_{0}$ and $m$ are unknown parameters. The method considers the joint density function for all observations. For an independent sample with known $\xi_{0}$ and $m$ likelihood, function

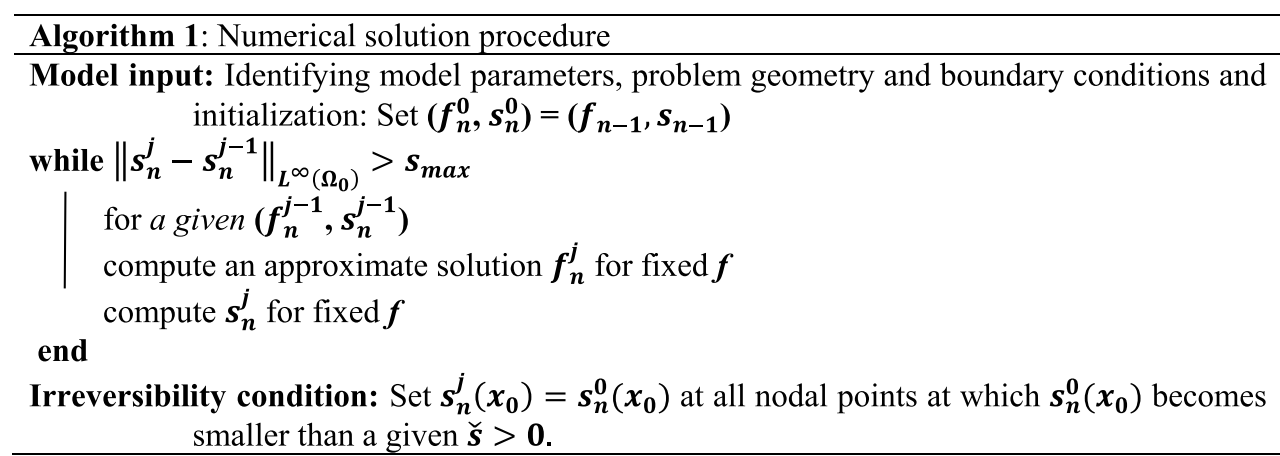

An adaptive $h$ refinement strategy (Del Piero et al. 2007) is used to automatically refine the elements with values of $s$ lower than the given thresholds. At new nodes generated through the remeshing strategy, the values of displacement and damage are calculated by linear interpolation from the existing nodes.
$L\left(\xi_{0}, m, \xi_{1}, \ldots \xi_{n}\right)$ is the joint density function of the $n$ random variables defined as

$$
L\left(\xi_{0}, m, \xi_{1}, \ldots \xi_{n}\right)=f\left(\xi_{1}, \xi_{2}, \ldots \xi_{n} \mid \xi_{0}, m\right)=\prod_{i=1}^{n} f_{x_{i}}\left(\xi_{i} \mid \xi_{0}, m\right)
$$




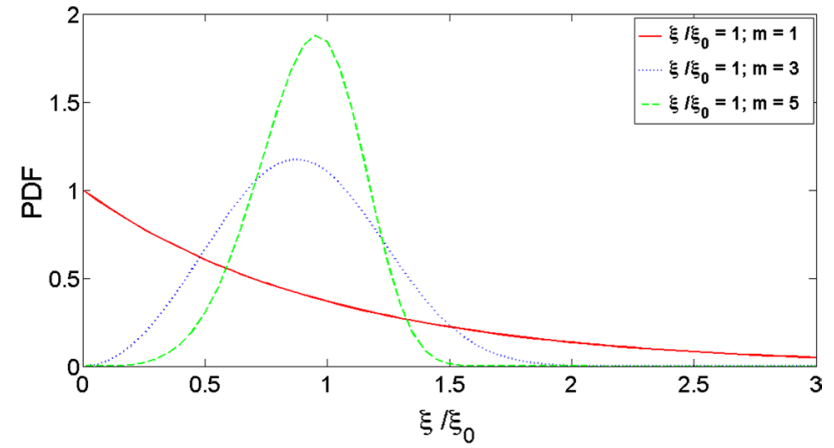

(a)

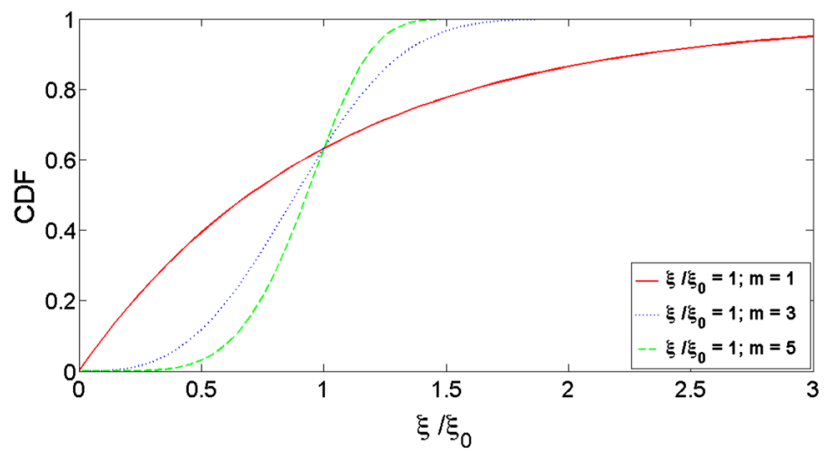

(b)

Fig. 2 Weibull function with different values of the shape parameter: a probability density function (PDF), and $\mathbf{b}$ cumulative distribution function (CDF)

On the basis of the MLE, the maximum likelihood of $\xi$ is achieved by maximizing $L$ or, for convenience, its logarithm

$\frac{\mathrm{d} \log L}{\mathrm{~d} m}=0, \quad \frac{\mathrm{d} \log L}{\mathrm{~d} \xi_{0}}=0$.

In order to apply the MLE to estimate the Weibull parameters, we can substitute Eq. (17) into Eq. (18) and apply Eq. (19); after a straightforward calculation one gets the following pair of equations

$\frac{\sum_{i=1}^{N} \ln \left(\xi_{i}\right) \xi_{i}^{m}}{\sum_{i=1}^{N} \xi_{i}^{m}}-\frac{1}{N} \sum_{i=1}^{N} \ln \left(\xi_{i}\right)-\frac{1}{m}=0$

$\xi_{0}=\left(\frac{\sum_{i=1}^{N} \xi_{i}^{m}}{N}\right)$

Equation (20a) is solved using the Newton-Raphson method to calculate the value of $m$ as

$m_{n+1}=m_{n}-\frac{f\left(m_{n}\right)}{f^{\prime}\left(m_{n}\right)}$

where $n$ is the Newton-Raphson iteration number, $f\left(m_{n}\right)=\sum_{i=1}^{n} \xi_{i}^{m} \ln \left(\xi_{i}\right) / \sum_{i=1}^{N} \xi_{i}^{m}-\frac{1}{m}-\frac{1}{N} \sum_{i=1}^{N} \ln \left(\xi_{i}\right)$

and

$$
\begin{aligned}
f^{\prime}\left(m_{n}\right)= & \sum_{i=1}^{n} \xi_{i}^{m}\left(\ln \xi_{i}\right)^{2}-\frac{1}{m^{2}} \sum_{i=1}^{n} \xi_{i}^{m}\left(m \ln \left(\xi_{i}\right)-1\right) \\
& -\left(\frac{1}{N} \sum_{i=1}^{N} \ln \left(\xi_{i}\right)\right)\left(\frac{1}{N} \sum_{i=1}^{n} \xi_{i}^{m} \ln \left(\xi_{i}\right)\right)
\end{aligned}
$$

The calculated value for $m$ is substituted into Eq. (20b) in order to obtain the scale parameter $\xi_{0}$ and define the Weibull distribution. Then, the inverse cumulative distribution function is used to generate random realizations over the simulation domain that is derived as

$P^{-1}(R)=\xi_{i}=-\xi_{0} \sqrt[m]{\ln (1-\Re)}$

where $\mathfrak{R}$ is a random number between 0 and 1 representing the probability of an occurrence. The full stochastic procedure is summarized in Algorithm 2.

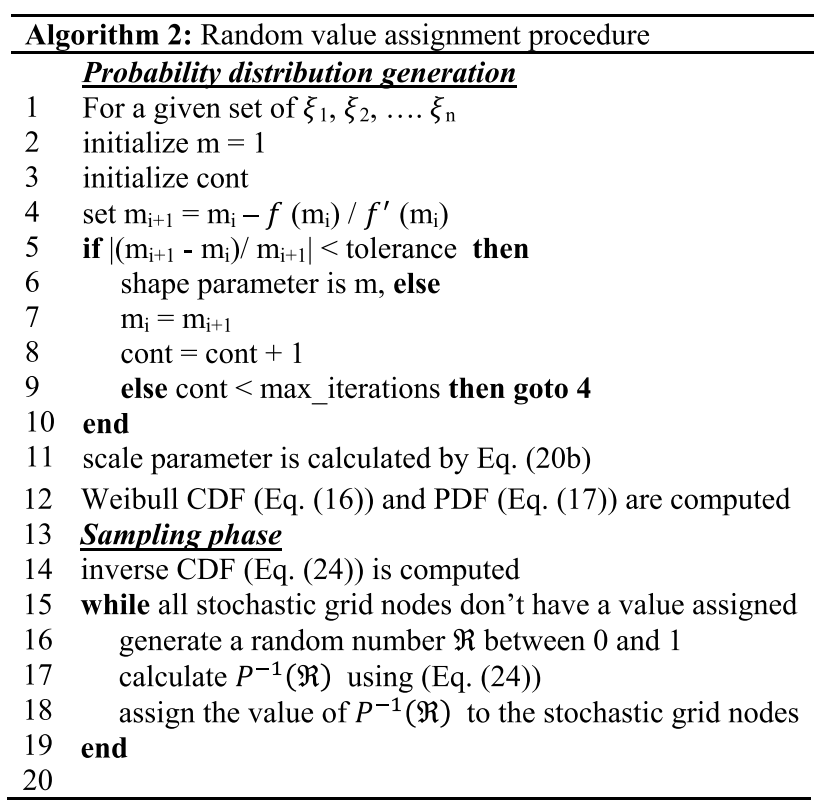

The scale parameter can be related to the size of the elements forming the material. It has been shown in Guy et al. (2012) that, for a specific range of material length scale and crack length over a domain, the material length scale itself can be used as scale parameter of the Weibull distribution. In our work, the material length scale is accounted in the generation of the hypothetical sample used successively to generate the shape and scale parameters by means of the MLE method. In this way, material length scale has been implicitly taken into account in the model. 


\section{Laboratory Tests Over Shale Rocks}

Theoretical equations to assess the strength of rocks, which are mainly based on the assumptions of isotropy and homogeneity, are not applicable for sedimentary rocks. Therefore, it is necessary to perform experimental studies to directly measure the mechanical properties of the shale samples. These experimental studies were conducted to investigate

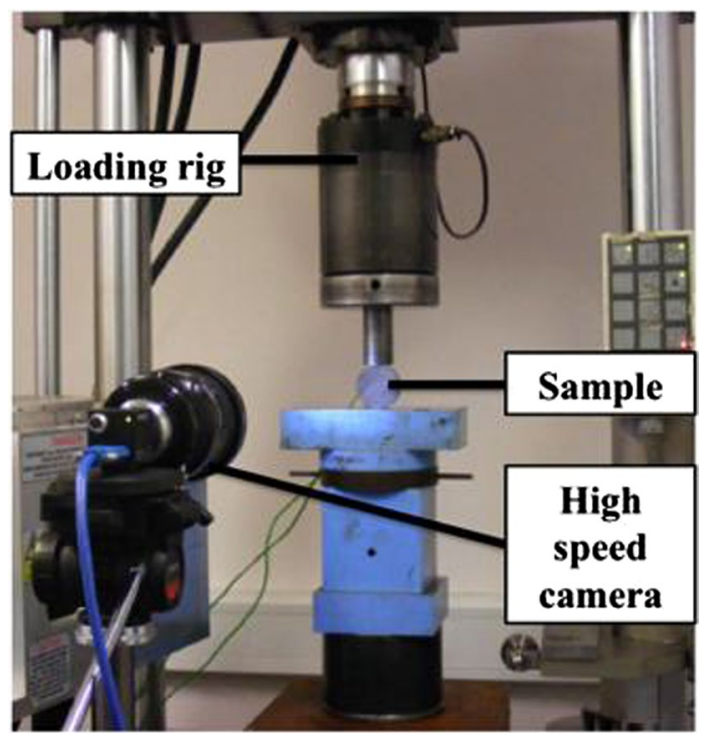

Fig. 3 Experimental setup in the current study

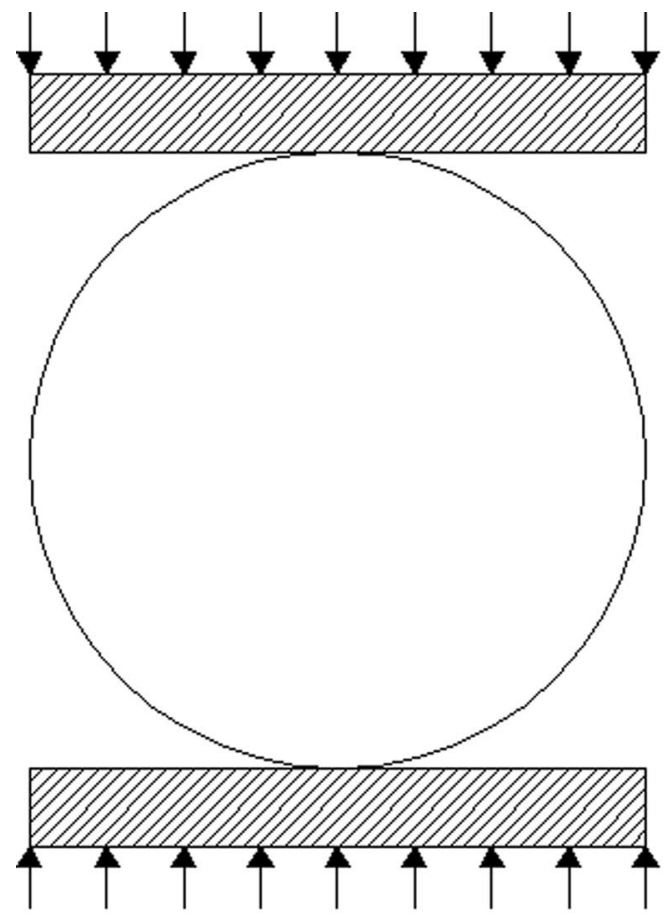

(a) the failure behavior of the shale rocks under compressive pressure, and their mechanical properties were measured. Then, the experimental data have been used to verify the proposed computational method and to identify the influence of factors such as heterogeneities and isotropy on both fracture propagation and strength.

\subsection{Experimental Setup}

The Brazilian tests, used to evaluate the tensile strength, were conducted on cylindrical samples taken from a depth of $246 \mathrm{~m}$. The experimental setup is shown in Fig. 3. The testing equipment consisted of a high-speed camera to take pictures of the fracture propagation, a strain gauge and a loading rig.

As loading condition significantly influences damage and stress distribution inside the specimen, following the typical methodology of the Brazilian tests, the loads were applied to the specimens in two different ways: (1) with flat platens and (2) with curved jaws (Fig. 4). Failure initiates directly under loading points if flat steel plates are used, and the specimen crack initiates at the center of the disk if curved jaws are employed (Hobbs 1964).

\subsection{Experimental Method and Measurements}

The fracture tests were carried out on specimens with different angles between loading direction and bedding plane

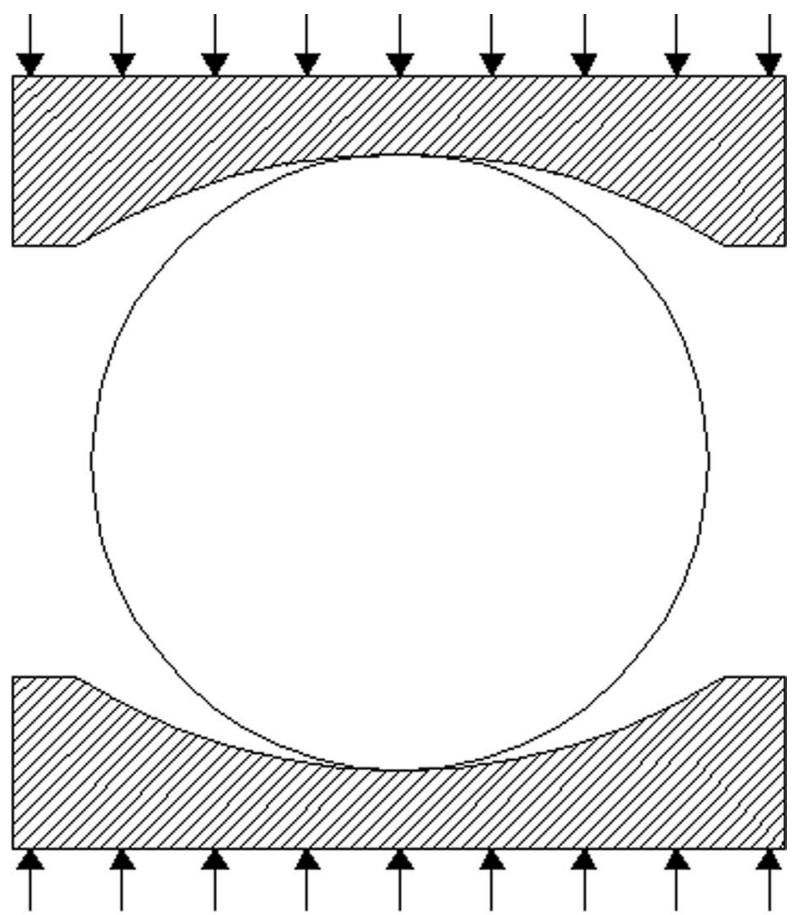

(b)

Fig. 4 Schematics of Brazilian disk test configurations, a with flat plates, and $\mathbf{b}$ with curved jaws 
orientations. The mechanical strength was determined by crushing the cylindrical specimens between steel plates. The variations of load, axial displacements and time were recorded from measurement devices connected to the loading frame (e.g., load cell and linear variable differential transformer or LVDT). Details of different testing specimens and measured data are presented in Tables 1 and 2 . For all specimens, fracture toughness is calculated using the method proposed in Guo et al. (1993). This method relies on the value of $P_{\min }$, which is the post-residual load being maintained by each fractured disk. The elastic modulus, $E$, was determined by attaching an electrical resistance strain gauge at the center of a disk to measure the lateral strains until failure.

All test specimens are shown in Fig. 5; they were all tested in an air-dried state. In this figure it can be observed that all specimens had a well-defined layered structure, some with rather visible inclusions (e.g., in specimens $\mathrm{C}$ and D).

The indirect tensile strength $\left(\sigma_{t}\right)$ of the cylindrical specimen under compression along the vertical diameter of the specimen was obtained using ( $\mathrm{Li}$ and Wong 2013)

$\sigma_{t}=\frac{P_{\max }}{\pi R t}$

where $P_{\max }$ is the maximum applied load to the specimen before failure, $R$ is the radius of the specimen, and $t$ is its thickness. The tensile stress-strain plots from tests on all specimens are shown in Fig. 6. For the case of experiments conducted using flat plates, specimen B showed less stiffness, but a higher value of ultimate tensile strength and a wider post-elastic behavior. For the experiments conducted with curved jaws, the higher value of resistance was obtained
Table 1 Details of testing specimens and measured data using flat steel plates

\begin{tabular}{llllllllll}
\hline & $R(\mathrm{~mm})$ & $t(\mathrm{~mm})$ & $P_{\max }(\mathrm{KN})$ & $P_{\min }(\mathrm{KN})$ & $\sigma_{t}(\mathrm{MPa})$ & $E(\mathrm{GPa})$ & $v$ & $K_{I C}\left(\mathrm{MPa} \mathrm{m}{ }^{0.5}\right)$ & $G(\mathrm{MPa} \mathrm{m})$ \\
\hline A & 18.29 & 18.76 & 6.86 & 2.00 & 6.36 & 29.6 & 0.25 & 0.38 & $0.4 \times 10^{-2}$ \\
B & 18.30 & 20.69 & 9.46 & 4.22 & 8.04 & 18.5 & 0.25 & 0.72 & $2.6 \times 10^{-2}$ \\
\hline
\end{tabular}

\begin{tabular}{llllllllll}
\hline & $R(\mathrm{~mm})$ & $t(\mathrm{~mm})$ & $P_{\max }(\mathrm{KN})$ & $P_{\min }(\mathrm{KN})$ & $\sigma_{t}(\mathrm{MPa})$ & $E(\mathrm{GPa})$ & $v$ & $K_{I C}\left(\mathrm{MPa} \mathrm{m}^{0.5}\right)$ & $G(\mathrm{MPa} \mathrm{m})$ \\
\hline $\mathrm{C}$ & 23.7 & 21.87 & 11.90 & 4.85 & 7.3 & 26.6 & 0.25 & 0.6 & $1.5 \times 10^{-2}$ \\
$\mathrm{D}$ & 27.3 & 22.28 & 22.28 & 5.59 & 10.4 & 20.3 & 0.25 & 0.7 & $2.2 \times 10^{-2}$ \\
$\mathrm{E}$ & 30.81 & 27.26 & 29.25 & 14.29 & 11.2 & 24.0 & 0.25 & 1.38 & $7.5 \times 10^{-2}$ \\
\hline
\end{tabular}

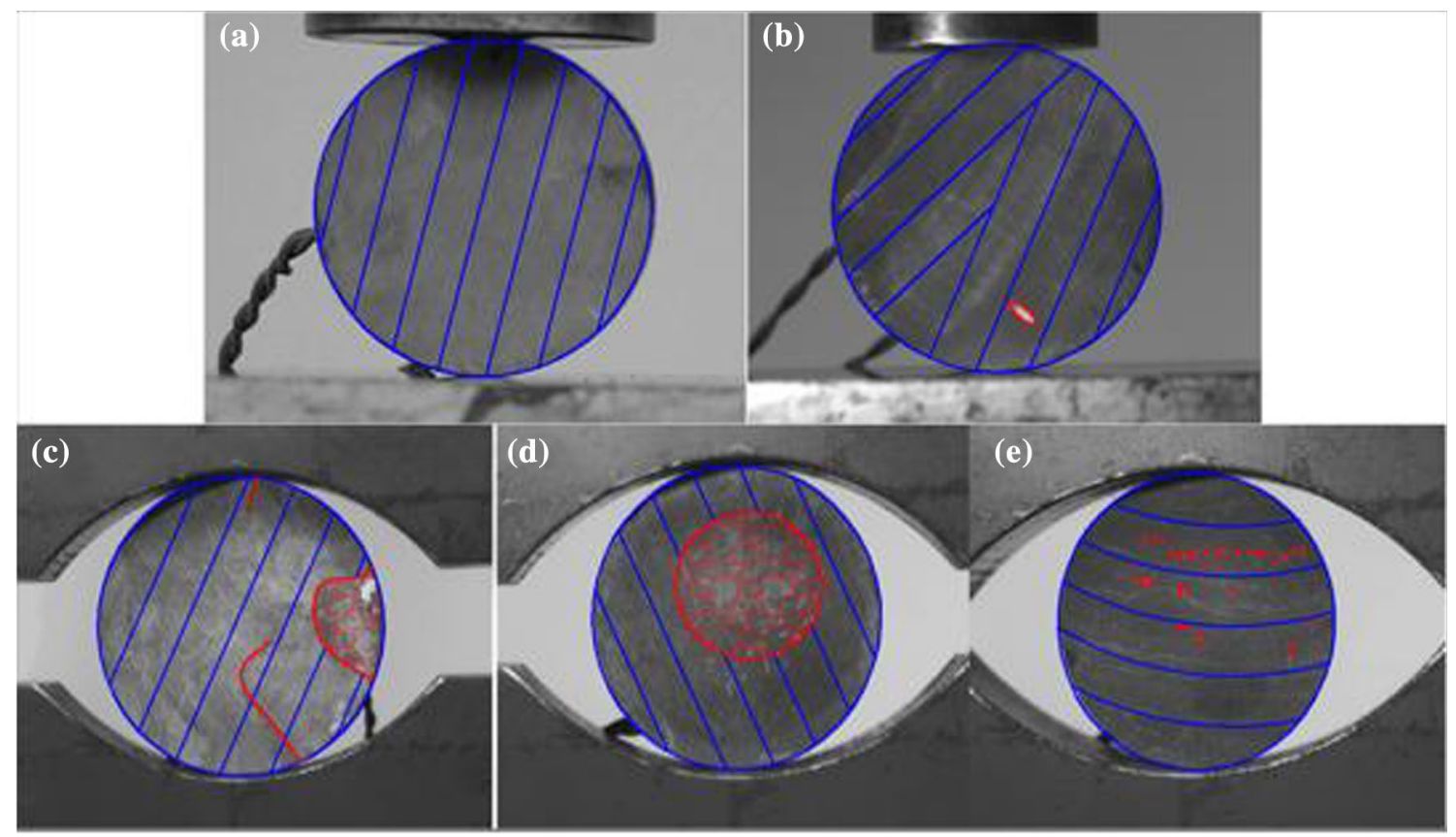

Table 2 Details of testing specimens and measured data using curved jaws 


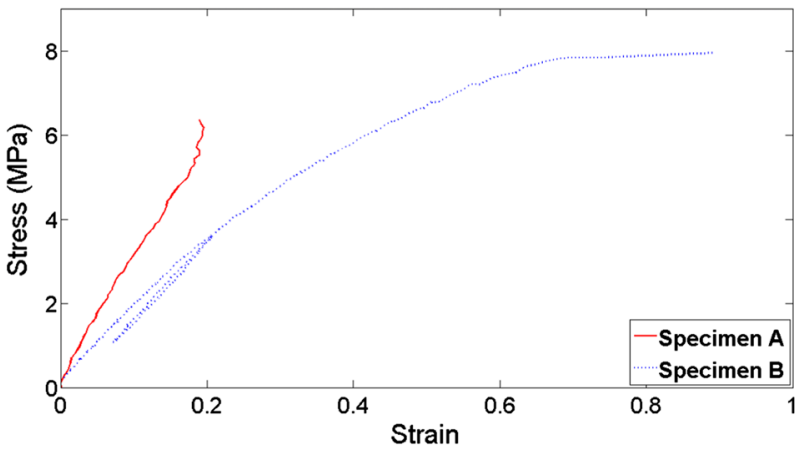

(a)

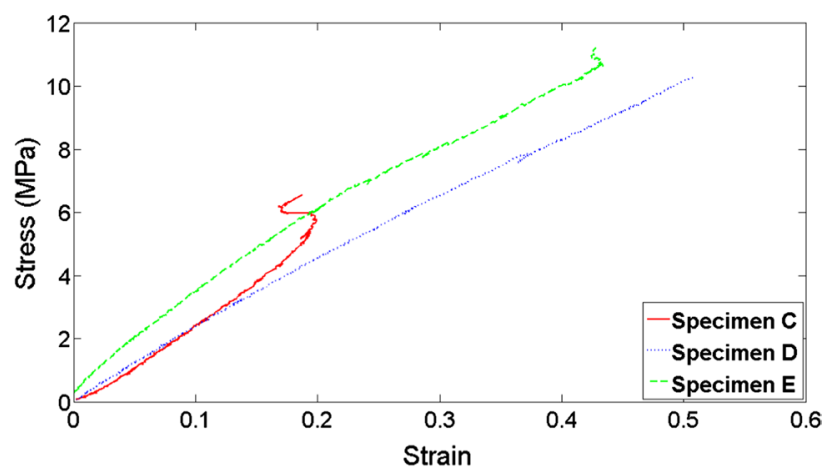

(b)

Fig. 6 Stress-strain plots from Brazilian disk tests on a specimens tested using flat plates, and $\mathbf{b}$ specimens tested using curved jaws for the specimen with a clear layered structure. To ensure the repeatability of the experiments, tests related to each case were repeated three times on specimens with similar characteristics and similar ranges for stress-strain variations have been observed for the test associated with each case. Therefore, the apparent differences among the plots presented in Fig. 6 can be associated with the variations of loading direction with respect to bedding direction.

Figure 7 shows all crack patterns obtained from the specimens described above. Crack pattern obtained for specimen A does not have a very noticeable tortuous nature. The specimen with a clear layered structure showed a well-defined crack pattern which was more curved compared to the one for specimen A. The specimens with less-defined layered structure and more scattered heterogeneous nature showed multiple and more tortuous cracks.

\subsection{Fracture Toughness Calculation}

As specified, for all of the specimens described above, fracture toughness was calculated using the method proposed in Guo et al. (1993). However, for specimen A the fracture toughness was calculated using a different methodology, given for this specimen $P_{\min }$ value was significantly low to be used for calculating a meaningful value of fracture toughness according to Guo et al. (1993). For this specimen it was decided to directly employ Irwin's equation, which describes the stress field distribution around the crack tip in
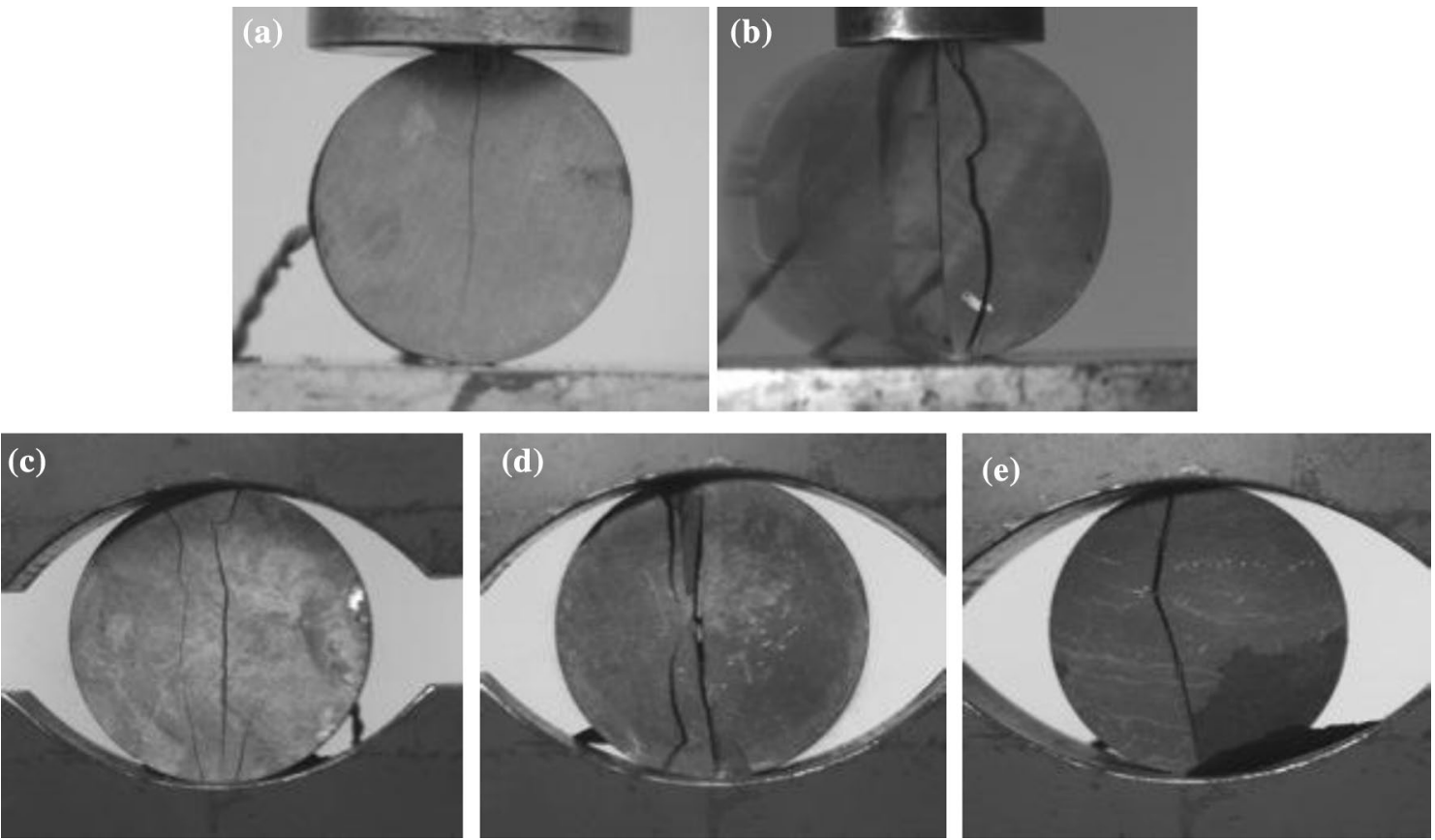

Fig. 7 Tested shale specimens: crack patterns obtained from specimens $\mathbf{a}, \mathbf{b}, \mathbf{c}, \mathbf{d}$ and $\mathbf{e}$ 
the two-dimensional polar coordinates as a linear function of the mode-I and mode-II stress intensity factors as in

$$
\begin{aligned}
\sigma_{\vartheta \vartheta}= & \frac{K_{\mathrm{I}}}{\sqrt{2 \pi r}}\left[\cos \left(\frac{\vartheta}{2}\right)\left[1-\sin \left(\frac{\vartheta}{2}\right) \sin \left(\frac{3 \vartheta}{2}\right)\right]\right] \\
& +\frac{K_{\mathrm{II}}}{\sqrt{2 \pi r}}\left[-\sin \left(\frac{\vartheta}{2}\right)\left[2+\cos \left(\frac{\vartheta}{2}\right) \cos \left(\frac{3 \vartheta}{2}\right)\right]\right]
\end{aligned}
$$

where $K_{\mathrm{I}}$ is mode-I stress intensity factor, $K_{\mathrm{II}}$ is mode-II stress intensity factor, and $r$ and $\vartheta$ are the crack tip polar coordinates.

The stress intensity factors are function of the initial crack length and locations (Knott and Elliott 1979); for the specimens without a manually created initial notch, spotting the location of the crack initiation point is crucial. To determine the crack initiation location, we followed the generalized maximum tangential stress (GMTS) criterion. The GMTS has been proved to be particularly suitable for rock-like materials (Aliha et al. 2010) and assumes that crack initiates when along the direction of maximum tangential stress and at a critical distance from the crack tip, $r_{c}$, the tangential stress, $\sigma_{\vartheta \vartheta}$, reaches to its critical value.

The location of maximum tensile strain point corresponds to the location of the transition point between a local shear damage zone in proximity of the loading points and tensile failure point (Fig. 8) (Hobbs 1964). Figure 9 shows the location of this damaged zone in specimen A. This local damage has spread over a distance of about $2.9 \mathrm{~mm}$, which approximately corresponds to $r_{c} / R \approx 0.84, r_{c}$ being the distance of the point from the disk center. This is in agreement with the finding of Hudson et al. (1972) that, using experimental observation and theoretical calculations, proved that the

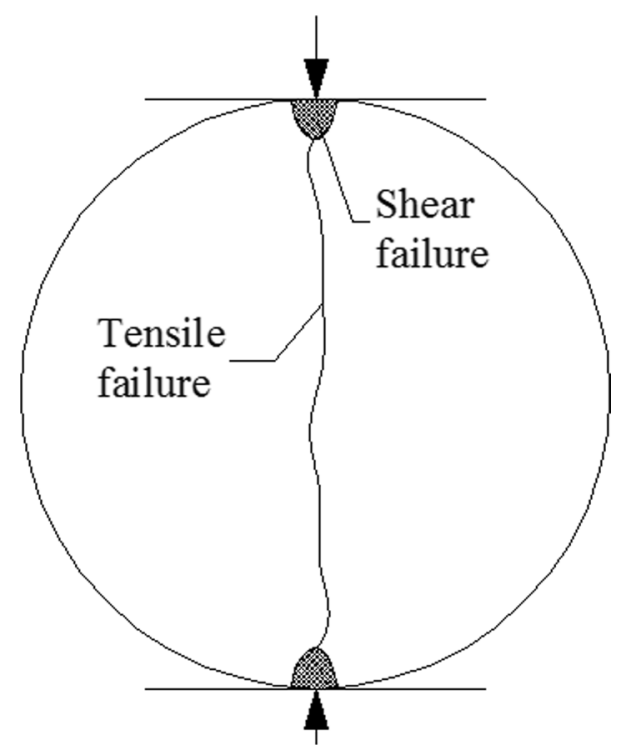

Fig. 8 Brazilian disk test failure modes according to Hobbs (1964) showing the transition between shear and tensile fracture zone crack initiation point (i.e., the point of the maximum radial and transversal strains) is located where $r_{c} / R$ is approximately between 0.8 and 0.9 .

Following the calculation of fracture toughness, fracture energy was calculated using (Knott and Elliott 1979)

$G=\frac{K_{I C}^{2}\left(1-v^{2}\right)}{E}$

where $E$ is the elastic modulus, $v$ is Poisson's ratio, and $K$ is the fracture toughness shown in Tables 1 and 2.

The values of the fracture toughness for all the other specimens were also calculated with the Irwin equation, and maximum and minimum values of $K_{I C}$ (and $G$ ) are reported in Table 3. In this table, the calculated values are also compared with those values obtained from the method based on theories proposed by Guo et al. (1993) and Hobbs (1964). The maximum and minimum values were evaluated considering two different distances between loading point and the crack initiation point. These two values have been chosen in such a way that $r_{c} / R$ lies between 0.85 and 0.95 .

Figure 10 plots the values of fracture toughness versus the material tensile strength. It can be observed that the proposed method is able to provide a range of values very close to the values of fracture toughness calculated using the method proposed by Guo et al. (1993). It is important to consider that the calculated value of the fracture toughness is a function of the position of the crack initiation point. When comparing these values, it should be considered that the observation of Hobbs (1964) is valid for specimens tested using flat plates. When curved jaws are used, the crack initiation point is usually more centrally located, this explains why for both $\mathrm{C}$ and $\mathrm{D}$ specimens the values calculated based on the method of Guo et al. (1993) are slightly out of the range of values calculated using Irwin's equation.

It is noticeable that according to finding of Ewy (2015), the values of mechanical parameters for shale-type specimens are influenced by the experimental conditions as well as the physical state of the specimens such as their saturation state. In this work, we used air-dried specimens assuming that they are in the same saturation condition and we have left the investigation of the effects of the saturation state of the specimens on their failure behavior to future works.

\section{Modeling and Numerical Analysis}

\subsection{Numerical Verification}

The experimental tests are modeled numerically using the developed computational framework. For the case of the homogeneous body, the geometry of the specimen is discretized using triangular elements. The mesh consists of 
Fig. 9 Local damage zone under loading point of specimen A, damage in the specimen and distance from the center of the disk

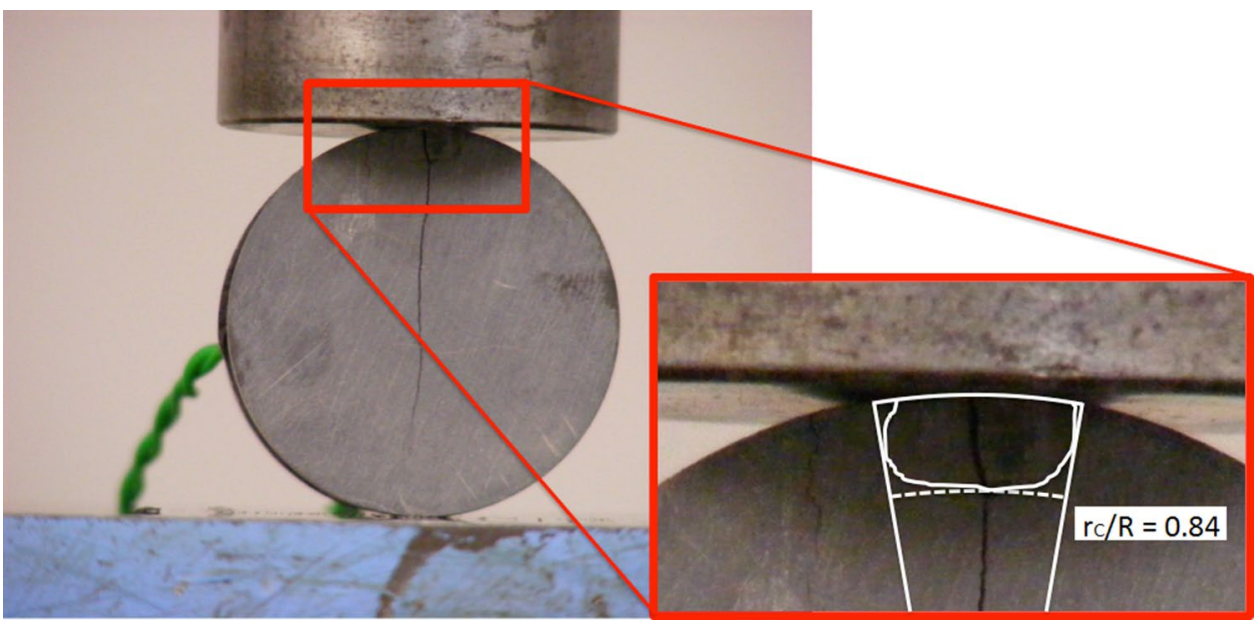

Table 3 Comparison between values of $K_{I C}$ (and $G$ ) calculated using the two different methods of Guo et al. (1993) and Irwin's equations

\begin{tabular}{|c|c|c|c|c|c|c|c|c|}
\hline & \multicolumn{2}{|l|}{ Guo et al. (1993) } & \multicolumn{6}{|c|}{ Irwin equation for fracture toughness combined with Hobbs' (1964) theory } \\
\hline & $K_{I C}\left(\mathrm{MPa} \mathrm{m}^{0.5}\right)$ & $G(\mathrm{MPa} \mathrm{m})$ & $r_{c} / R_{\min }$ & $\begin{array}{l}K_{I C \min }(\mathrm{MPa} \\
\left.\mathrm{m}^{0.5}\right)\end{array}$ & $G_{\min }(\mathrm{MPa} \mathrm{m})$ & $r_{c} / R_{\max }$ & $\begin{array}{l}K_{I C \max }(\mathrm{MPa} \\
\left.\mathrm{m}^{0.5}\right)\end{array}$ & $G_{\max }(\mathrm{MPa} \mathrm{m})$ \\
\hline A & 0.38 & $0.4 \times 10^{-2}$ & \multirow[t]{5}{*}{0.95} & 0.48 & $0.7 \times 10^{-2}$ & \multirow[t]{5}{*}{0.85} & 0.83 & $2.5 \times 10^{-2}$ \\
\hline B & 0.72 & $2.6 \times 10^{-2}$ & & 0.60 & $1.8 \times 10^{-2}$ & & 1.05 & $5.6 \times 10^{-2}$ \\
\hline $\mathrm{C}$ & 0.6 & $1.5 \times 10^{-2}$ & & 0.62 & $1.3 \times 10^{-2}$ & & 1.11 & $4.1 \times 10^{-2}$ \\
\hline $\mathrm{D}$ & 0.7 & $2.2 \times 10^{-2}$ & & 0.96 & $4.2 \times 10^{-2}$ & & 1.66 & $1.2 \times 10^{-1}$ \\
\hline $\mathrm{E}$ & 1.38 & $7.5 \times 10^{-2}$ & & 1.10 & $4.7 \times 10^{-2}$ & & 1.9 & $1.4 \times 10^{-1}$ \\
\hline
\end{tabular}

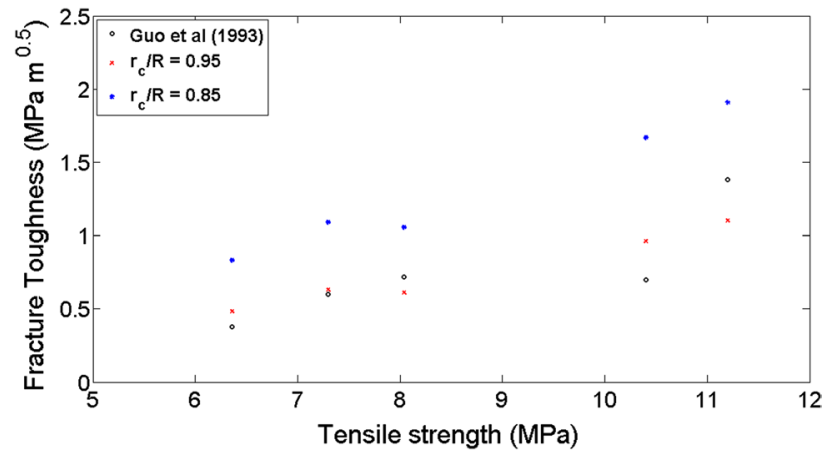

Fig. 10 Comparison between the values of fracture toughness calculated with the two methods considered in this work

1024 elements and 551 nodes (Fig. 11a). Figure 11b shows a typical comparison between the numerically predicted and experimentally observed crack paths. Except for some small deviations, the path obtained from numerical simulation is in good agreement with the one observed from the experiment. The deviations can be due to factors such as shear effects and load concentrations (Hobbs 1964).

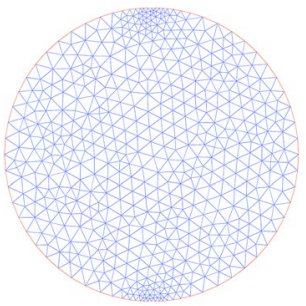

(a)

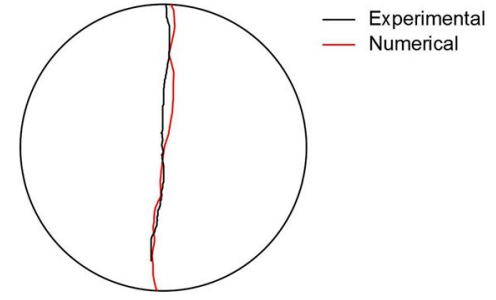

(b)
Fig. 11 a Two-dimensional finite element mesh of the Brazilian disk, and $\mathbf{b}$ comparison between experimentally observed and numerically predicted crack paths

The color map in Fig. 12 provides information regarding the evolution of fracture parameter $s$ on the simulation domain, describing the evolution of the damage state of the specimen. Under the loading point, a local damage zone is formed; this is the zone where failure initiates. Figure 13 shows the evolution of the total, bulk and fracture energies with time, which indicates the onset of the failure. It is noticeable that the highest concentration of damage is located not immediately under the loading point, but at a 


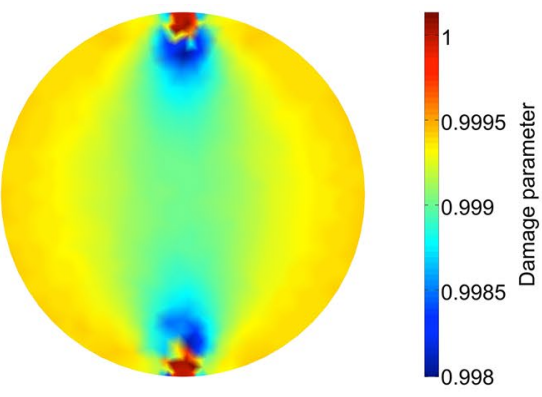

(a)

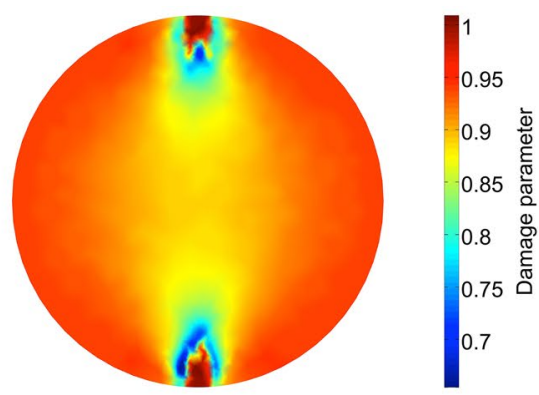

(b)

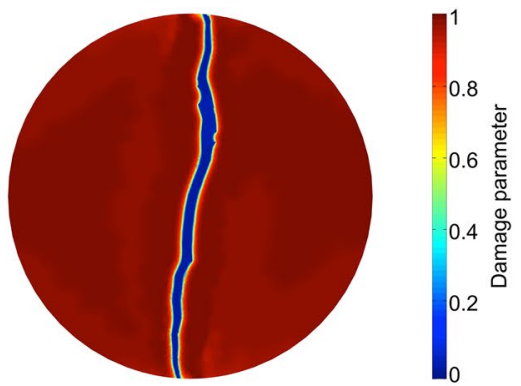

(c)

Fig. 12 Damage state evolution in the disk at different time steps for specimen A, a after one load step, b before failure and $\mathbf{c}$ after failure

distance of $2.9 \mathrm{~mm}$ from the loading point, compatible with conclusions from Hudson et al. (1972). Numerical simulations of a comparable test presented by Li and Wong (2013) also show that crack initiates at the same location, underneath the loading points, where maximum tensile stress is generated (see Fig. 13).

It can be clearly observed that all energies increase monotonically, but when rupture occurs, a noticeable decrease in the slope of the bulk energy appears, which deviates from the total energy; then microcracks are consequently forming and starting to coalesce. New crack surface is formed, leading to a consequent increase in the amount of fracture energy, proportional to crack surface. The deformation energy before reaching failure decreases as it is released and converted for the creation of the crack surface. Furthermore, in the instant immediately before failure, damage parameter $s$ reaches to its lowest value (about 0.65 ) in the zone closest to the loading plates.

In this study we followed indications given by $\mathrm{Li}$ and Wong (2013) who recalled the International Society of Rock Mechanics guidelines. A Brazilian test is usually executed

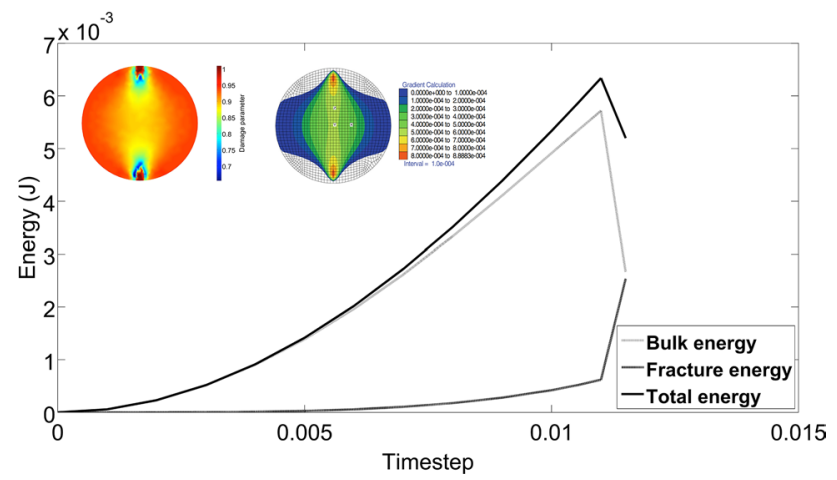

Fig. 13 Evolution of total, bulk and fracture energies as function of time, and damage state of the specimen in the instant before failure analyzed with both the numerical model developed in this work (left) and with FLAC3D 3.10 (right). (Reproduced with permission from $\mathrm{Li}$ and Wong 2013) creating a $5^{\circ}$ to $15^{\circ}$ contact arc between specimen and loading jaws which, for the case study considered in this work, leads to a length of between 3.2 and $1.5 \mathrm{~mm}$. For the simulations reported for the specimen A where the flat plates were used, we applied a distributed displacement over length equal to $2 \mathrm{~mm}$ at the two boundaries (above and below) of the disk. One analysis has been performed also for one of the specimens (specimen D) tested using curved jaws, and the result in terms of crack path is provided in Fig. 14. In this case, the specimen has a radius of $27.3 \mathrm{~mm}$, and the contact arc between specimen and curved jaws is taken equal to $5 \mathrm{~mm}$; the force applied on this arch is maximum on the middle-top point of the specimen and decreases for points distant from the middle point. In this work, a quasi-static fracture model is used for the simulation of fractures in Brazilian disks; a quasi-static modeling framework is known to be sufficient for prediction of multiple fractures in Brazilian disks (see Duan and Kwok 2015). However, simulation of crack branching in the shale rocks is still an open challenge, and we leave the extension of the current computational model for simulation of dynamic fracture propagation and modeling of the crack branching to the future work.

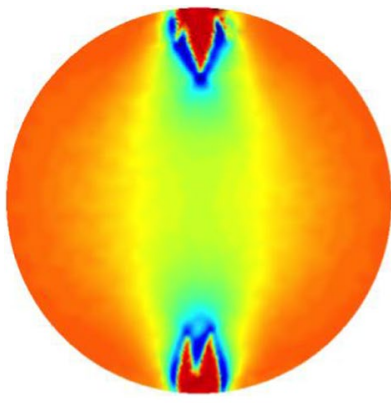

(a)

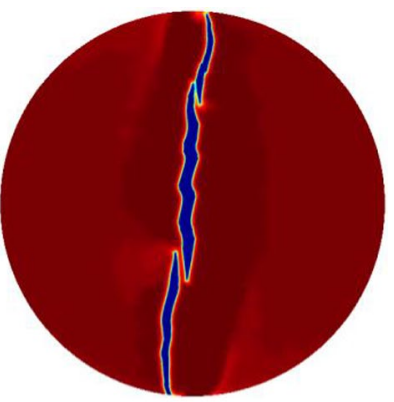

(b)
Fig. 14 Damage state evolution for specimen D, a before failure, and b after failure 


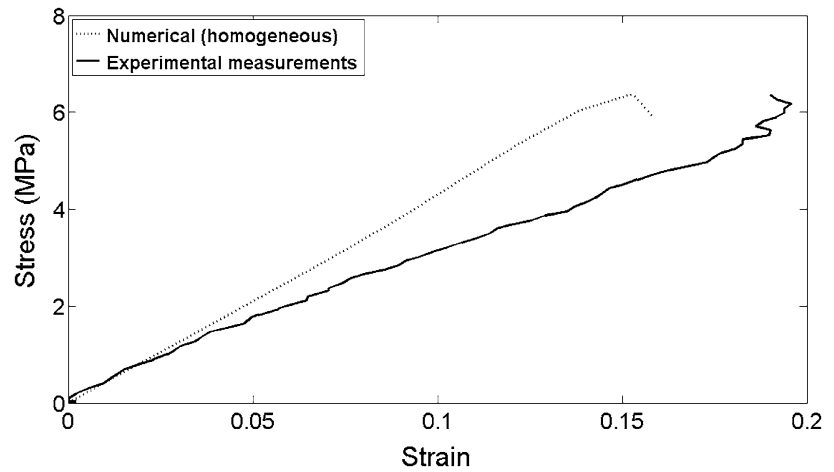

Fig. 15 Comparison between the experimentally measured and numerically simulated stress-strain plots for specimen A

Figure 15 shows the stress-strain plots at the center of the disk. Although for small values of strain the numerical predictions match well with the experimental data; however, a considerable discrepancy in the results is observed as strains increase. The tensile strength obtained from experimental test and numerical simulation is both equal to $6.36 \mathrm{MPa}$, but a smaller failure strain is predicted by the numerical simulation. These discrepancies may be due to the dissipation of energy associated with mesoscale cracking which is not considered in this simulation. In shale rocks, significant amount of energy is dissipated in the fracture process zone and in the vicinity of the crack front (Ding et al. 2012). In this simulation materials are considered to be fully brittle and the developed framework does not include a constitutive law for cohesive behavior of rock-like materials.

\subsection{Influence of Bedding Direction}

Investigating the influence of the rock bedding orientation on propagation of fractures allows determining the optimal angle at which cracks will grow in a desired length and direction. The anisotropic nature of the shale rock, in fact, creates huge differences in results when different bedding plane directions are considered (Morgan and Einstein 2014). We considered four distinct models labeled a, b, c and d (see Fig. 16) that differ by the angle between the direction of loading and the orientation of the bedding planes, at $90^{\circ}$, $60^{\circ}, 30^{\circ}$ and $0^{\circ}$, respectively. Different values of material
Table 4 Input mechanical properties for layered material models

\begin{tabular}{llll}
\hline Layer & $E(\mathrm{GPa})$ & Poisson's ratio $(v)$ & $\begin{array}{l}\text { Fracture } \\
\text { energy } \\
(\mathrm{MPa})\end{array}$ \\
\hline $\mathrm{A}$ & 25.1 & 0.22 & $3.0 \times 10^{-2}$ \\
$\mathrm{~B}$ & 28.1 & 0.24 & $2.7 \times 10^{-2}$ \\
$\mathrm{C}$ & 31.1 & 0.26 & $2.4 \times 10^{-2}$ \\
$\mathrm{D}$ & 34.1 & 0.28 & $2.1 \times 10^{-2}$ \\
\hline
\end{tabular}

properties are assigned to each layer, and they are all summarized in Table 4 . These values have been chosen in a way that their calculated mean value is equal to the single value obtained by the experimental measurements.

Crack paths for each layered model and comparison with damage state over the specimen are shown in Fig. 17. Crack paths are influenced in accordance with direction of bedding layers. The values for damage parameter are different in different layers, and bedding direction influences this damage localization. The variability of mechanical properties and damage state, which is not uniformly distributed over the specimen, influences the pattern of the cracks; in fact, cracks show different morphology, and for example observing the crack path obtained for the case with horizontal bedding, it can be seen that multiple cracks are found.

Stress-strain curves for different models are shown in Fig. 18. The values for maximum tensile strength for all models, except for the model with the $30^{\circ}$ bedding orientation, are higher than the one achieved from homogeneous simulation. Figure 19 shows how effectively tensile strength is the function of bedding angle, and that the highest value is found for the specimen with horizontal layers. The maximum value of tensile strength and higher stiffness is obtained for the specimen with vertical layers, and the lowest corresponding values are associated with the model with a $30^{\circ}$ bedding inclination. This behavior is also observed in the graphs of Fig. 6, in which specimen E, which has almost horizontal layers, has a higher stiffness and ultimate strength compared to other specimens. In order to further study the effects of anisotropic nature of shale formation, additional analyses were performed for different bedding orientations and different values of mechanical properties for each layer
Fig. 16 Bedding directions for Brazilian disk: bedding at a $90^{\circ}$, b $60^{\circ}$, c $30^{\circ}$ and $\mathbf{d} 0^{\circ}$

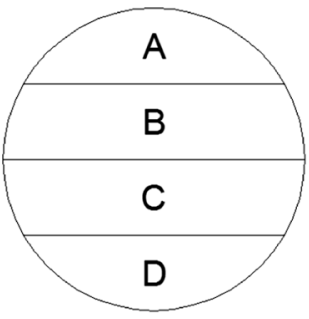

(a)

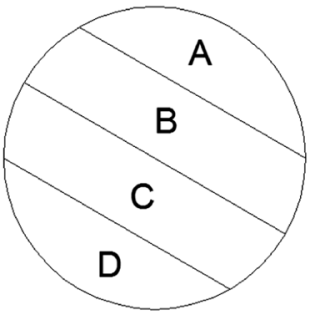

(b)

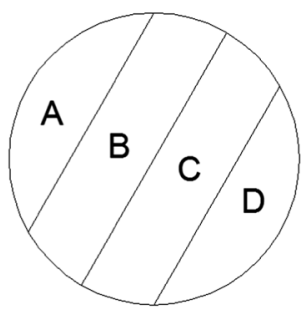

(c)

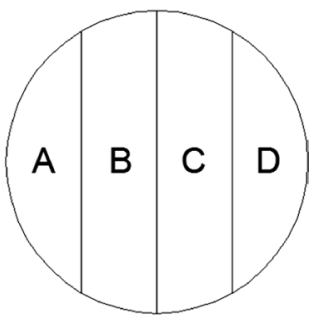

(d) 
Fig. 17 Crack paths and damage state for layered materials, bedding at a $90^{\circ}, \mathbf{b} 60^{\circ}$, c $30^{\circ}$ and $\mathbf{d} 0^{\circ}$

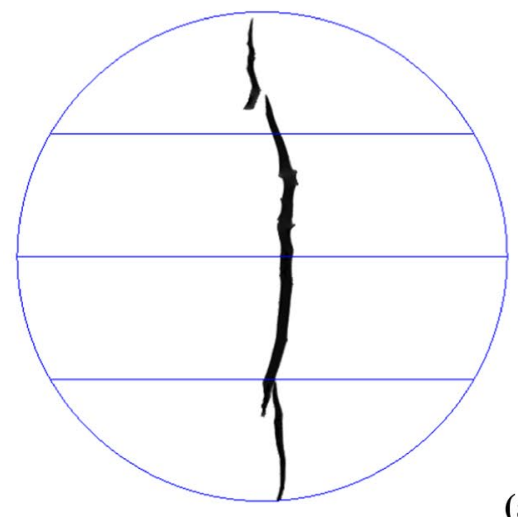

(a)
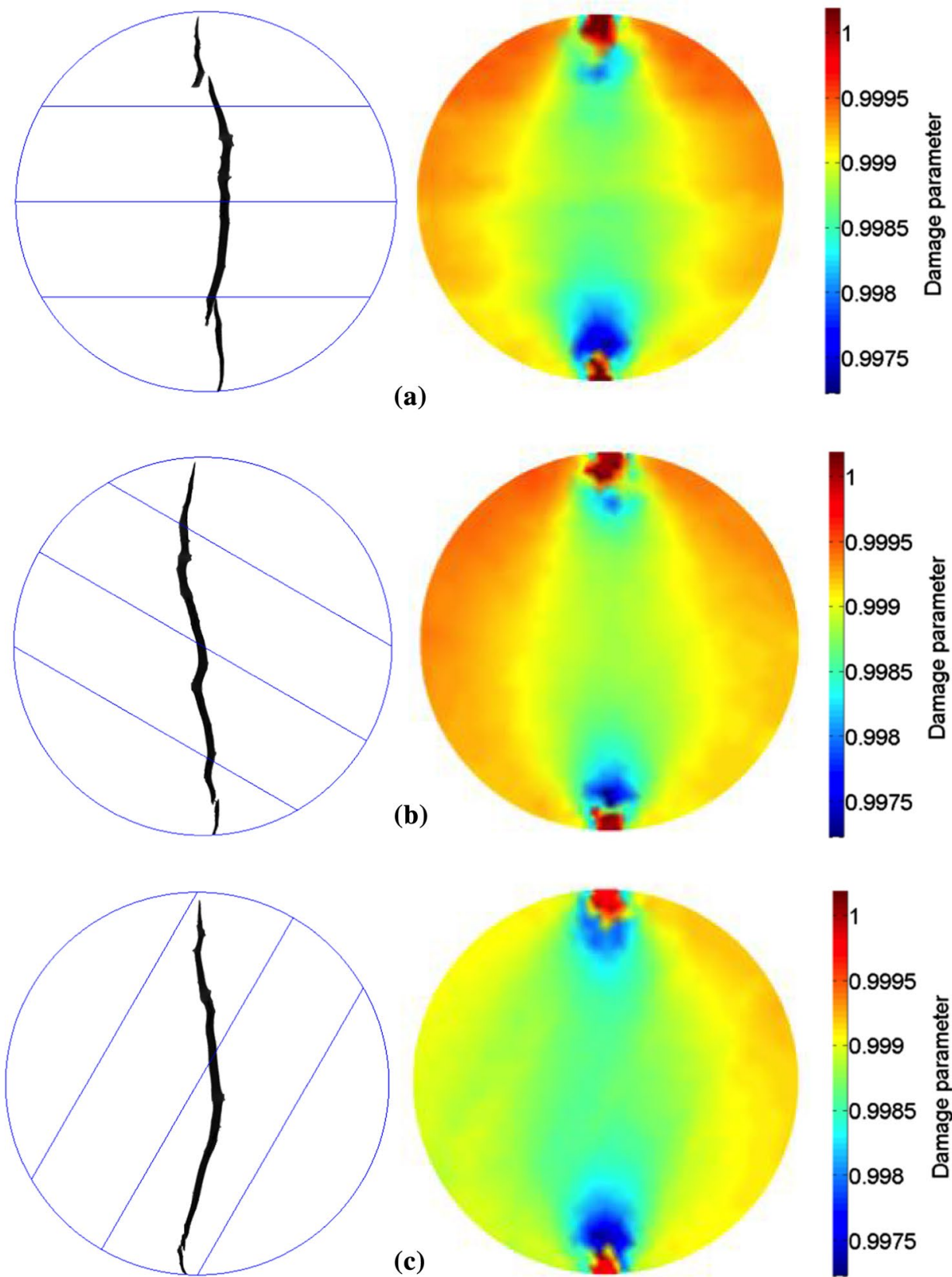

(c)
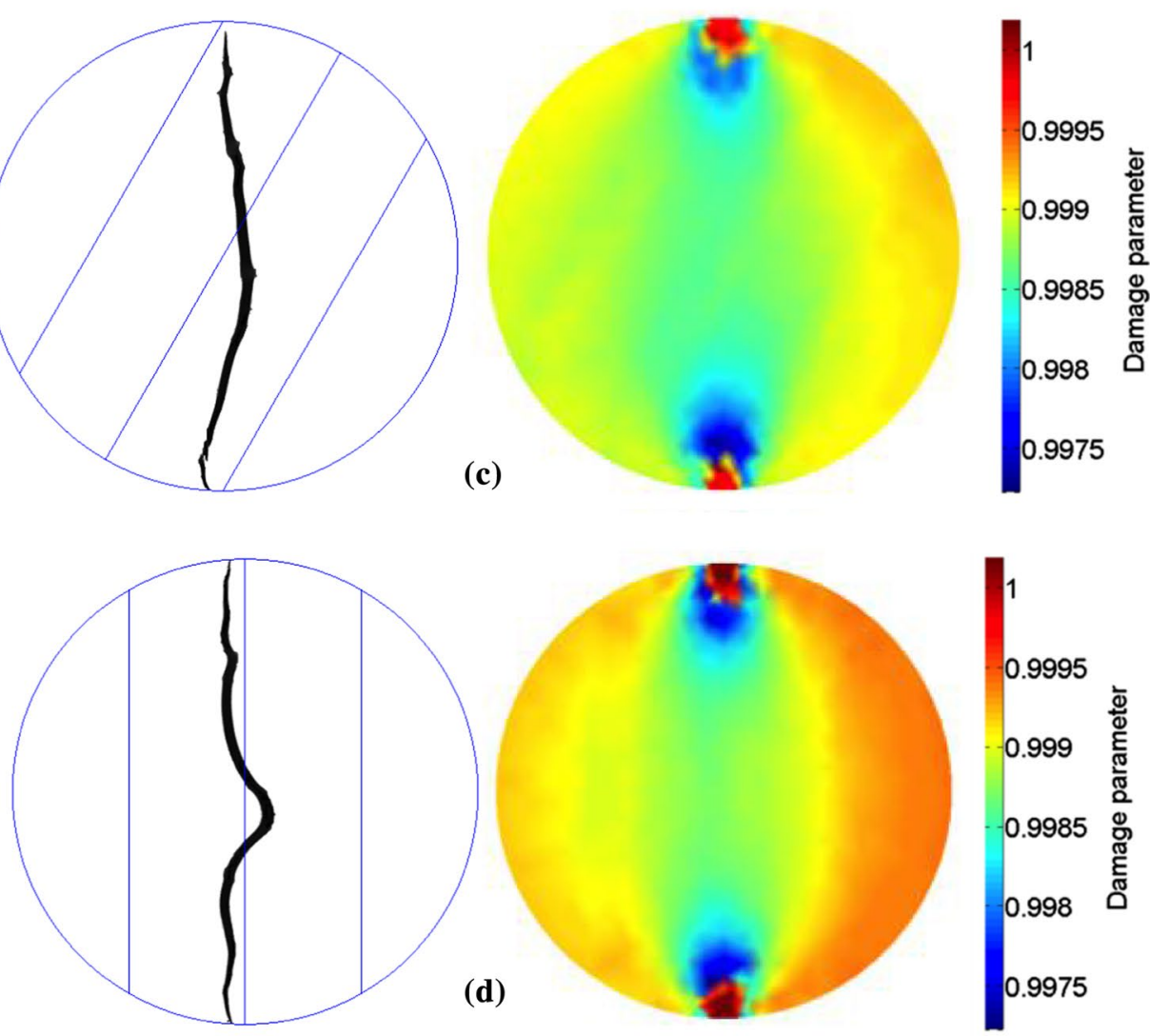

(d)

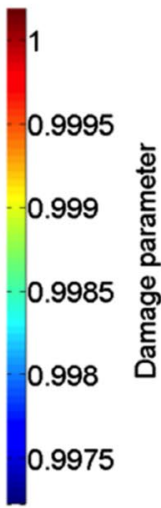




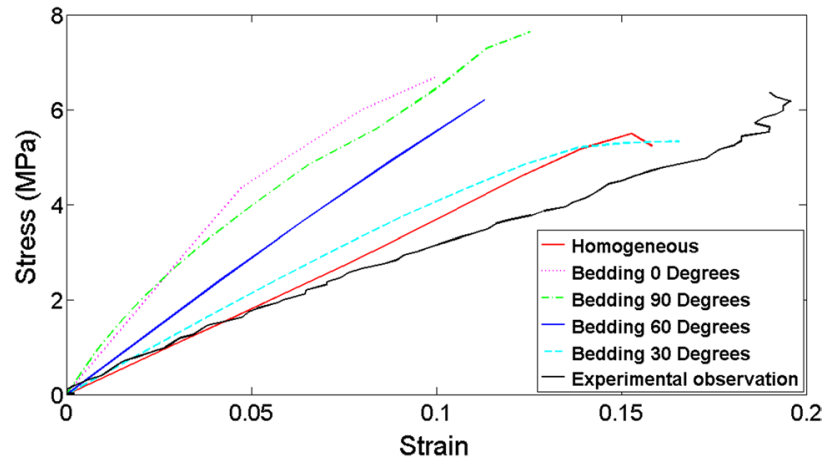

Fig. 18 Stress-strain curves for layered materials and comparison with homogeneous and layered models

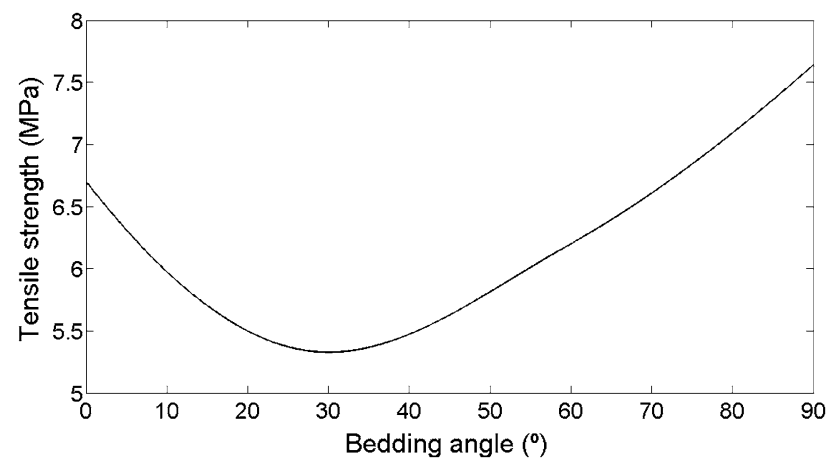

Fig. 19 Tensile strength as function of bedding direction

(see Table 5). Figure 20 shows all crack patterns obtained for these additional scenarios, for the same bedding directions as above, while Fig. 21 plots the tensile strength values for all scenarios, considered in this section, together with their mean curve. The average maximum tensile strength is observed for loading perpendicular to the bedding direction, and it decreases with the bedding direction angle.

\subsection{Spatial Variability}

In order to take into account the microscale material heterogeneity, a statistical technique is employed. The Weibull distribution function (Weibull 1939) is used to generate random

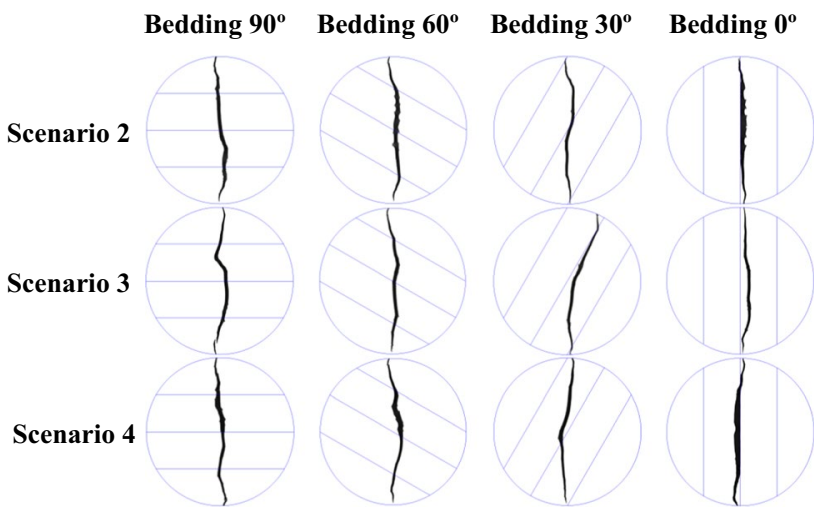

Fig. 20 Crack patterns obtained for the layered models with input values summarized in Table 5 , crack paths not only vary when the orientation of the layers changes, but also when different values of mechanical properties are applied to the same layers

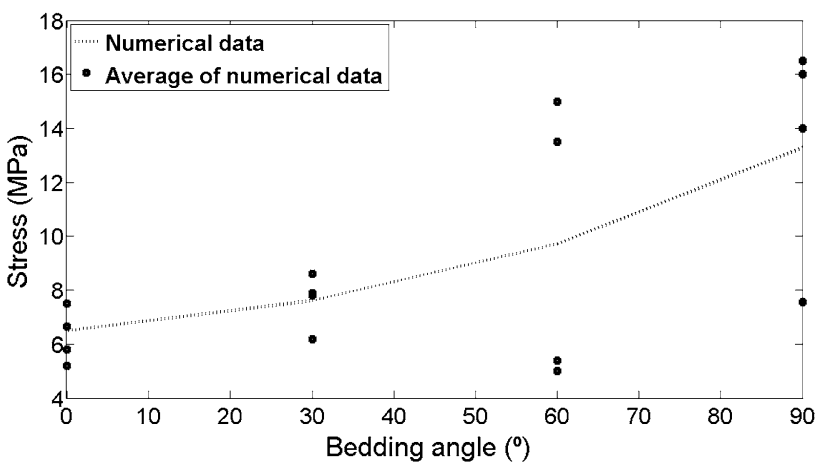

Fig. 21 Values of tensile stress obtained for all layered models considered in this study, together with their mean curve

distributions of the shale properties. In this study, we considered fracture energy as a random variable and Fig. 22 shows a typical realization of random fracture energy. The correlation length is kept equal to $1.0 \mathrm{~mm}$ for all the simulations, and two different degrees of standard deviation are considered equal to 1 and $10 \%$.

Crack path obtained for one of the models with heterogeneous distribution of the fracture energy (standard deviation
Table 5 Input mechanical properties for additional scenarios of layered models

\begin{tabular}{|c|c|c|c|c|c|c|c|c|c|}
\hline \multirow[t]{2}{*}{ Layer } & \multicolumn{3}{|c|}{ Scenario 2} & \multicolumn{3}{|c|}{ Scenario 3} & \multicolumn{3}{|c|}{ Scenario 4} \\
\hline & $E(\mathrm{GPa})$ & $v$ & $G(\mathrm{MPa} \mathrm{m})$ & $E(\mathrm{GPa})$ & $v$ & $G(\mathrm{MPa} \mathrm{m})$ & $E(\mathrm{GPa})$ & $v$ & $G(\mathrm{MPa} \mathrm{m})$ \\
\hline A & 30.1 & 0.26 & $2.6 \times 10^{-2}$ & 30.1 & 0.26 & $2.6 \times 10^{-2}$ & 31.1 & 0.26 & $2.4 \times 10^{-2}$ \\
\hline B & 28.1 & 0.24 & $2.4 \times 10^{-2}$ & 32.1 & 0.28 & $3.0 \times 10^{-2}$ & 34.1 & 0.28 & $2.1 \times 10^{-2}$ \\
\hline $\mathrm{C}$ & 26.1 & 0.22 & $2.2 \times 10^{-2}$ & 26.1 & 0.22 & $2.2 \times 10^{-2}$ & 25.1 & 0.22 & $3.0 \times 10^{-2}$ \\
\hline D & 32.1 & 0.28 & $3.0 \times 10^{-2}$ & 28.1 & 0.24 & $2.4 \times 10^{-2}$ & 28.1 & 0.24 & $2.7 \times 10^{-2}$ \\
\hline
\end{tabular}




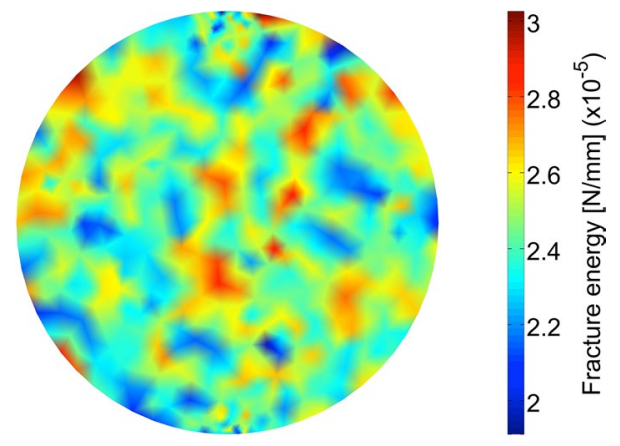

Fig. 22 Realization for random distribution of fracture energy in the Brazilian disk

10\%) is shown in Fig. 23 and is compared with the crack path obtained from the homogeneous model. It is observed

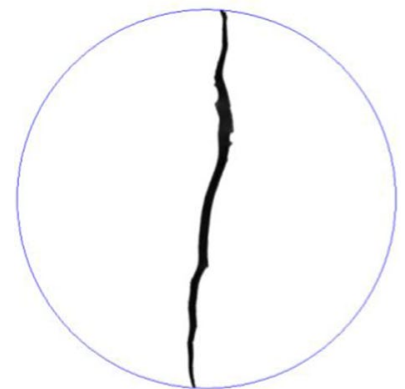

(a)

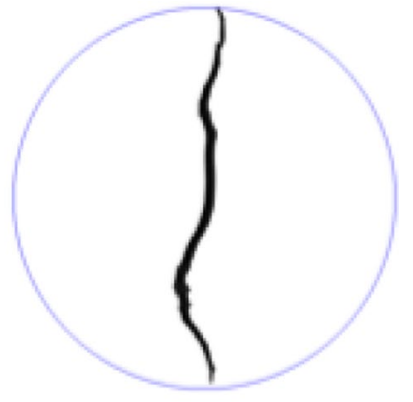

(b)
Fig. 23 Comparison between $\mathbf{a}$ homogeneous, and $\mathbf{b}$ one of the heterogeneous models

that crack path obtained from the model considering a heterogeneous material shows a more irregular pattern, as crack tends to propagate toward zones of less resistance. Figure 24 shows the evolution of the damage state of the body for both homogeneous and heterogeneous models. It shows that heterogeneity generates a random distribution of local damage zones in the specimen, which can significantly affect the mechanical characteristics of the body.

In Fig. 25 all of the stress-strain curves resulted from 100 simulations associated with different random realizations of fracture toughness and their mean curve are plotted and compared with the stress-strain curves obtained from experimental tests and numerical modeling considering the homogeneous model. The stochastic result shows a better agreement with experimental measurements particularly in terms of failure strain. Figure 26 summarizes the results obtained with different values of standard deviation, while Fig. 27 shows how convergence of the values of tensile strength increases with the number of simulations.

For all the correlation lengths investigated, results show that peak load decreases as the standard deviation increases. This is because larger areas in the specimen are fallen into the damage zone when the standard deviation is large, leading to a lower mean peak load. When the standard deviation is large, the weakest link or the crack path can be more easily found because more local damage zones will be generated and smaller zone of the specimen contributes to fracture resistance, which results in lower mean peak load. This may explain why a higher heterogeneity in the material properties results in structures with lower strength and why the numerical results with inclusion of heterogeneity get a better agreement with the experimental results.

\subsection{Layered Specimens with Spatial Variability Approach}

In order to consider the layered nature of the material, we apply the stochastic approach explained in the previous section (Sect. 4.3) considering as correlation length equal to the thickness of each layer. The correlation length defines how the variability is spatially distributed and can provide a realistic picture of the layers forming the samples. For this example, simulations were conducted by considering a correlation length of $10 \mathrm{~mm}$ and a standard deviation of $10 \%$.

Figure 28 shows the crack paths achieved through numerical simulation performed to demonstrate the effects of bedding directions on the direction of crack propagation. In these models fracture energy changes across the layers, resembling a heterogeneous layered formation. Figure 29 compares the experimental crack pattern from specimen A with the numerical crack pattern obtained for a model with bedding inclination similar to that of specimen A. The figure shows similar shape and pattern of crack within the specimen and the model. Figure 30 compares the experimentally measured and numerically obtained plots of stress-strain. There is a good agreement between the experimental results and numerical predictions. However, comparing with the results presented in Fig. 25, it can be concluded that the nonlinear nature of the material behavior is not as well captured as when the heterogeneity is included by considering the spatial variability. 
Fig. 24 Damage state for homogeneous (left) and heterogeneous (right) models at different steps of the analysis
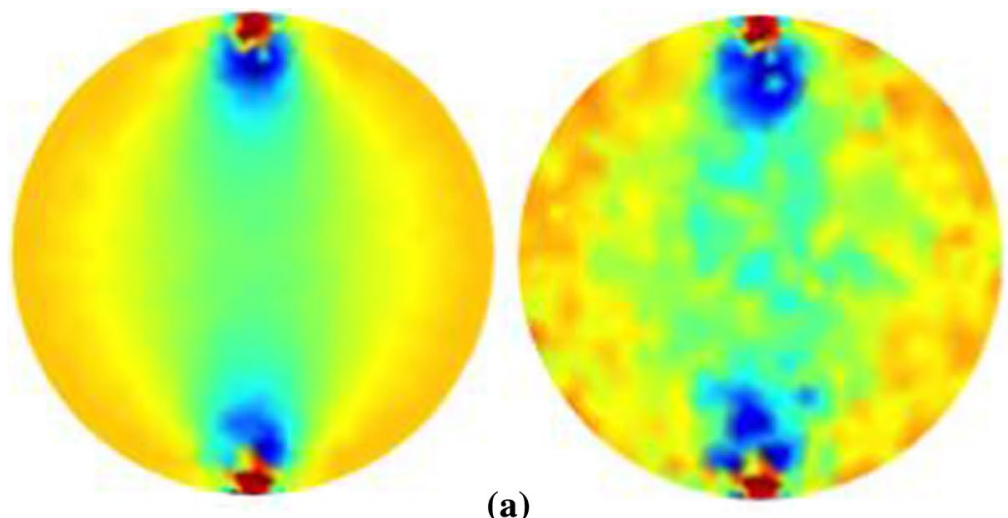

(a)
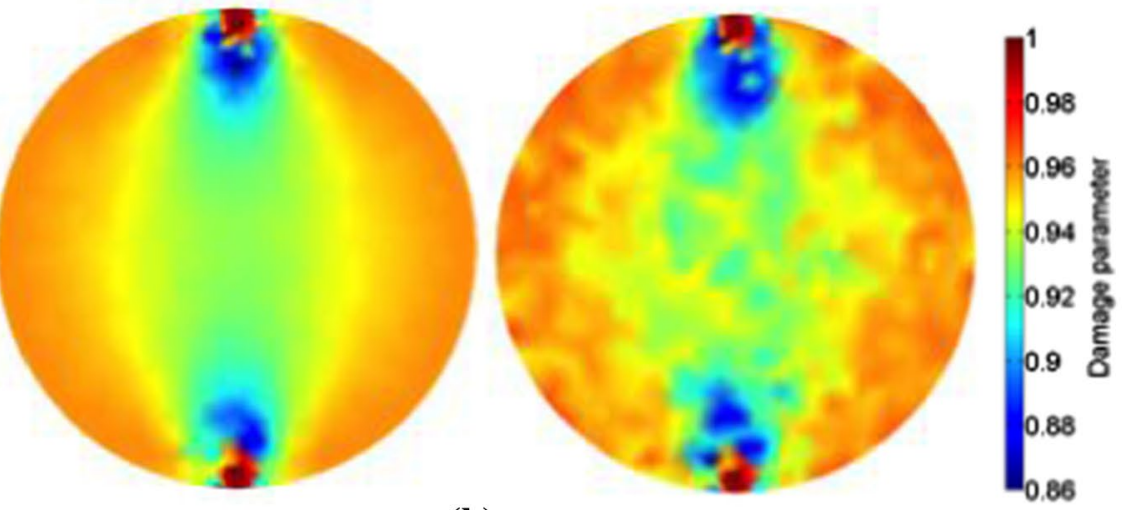

(b)
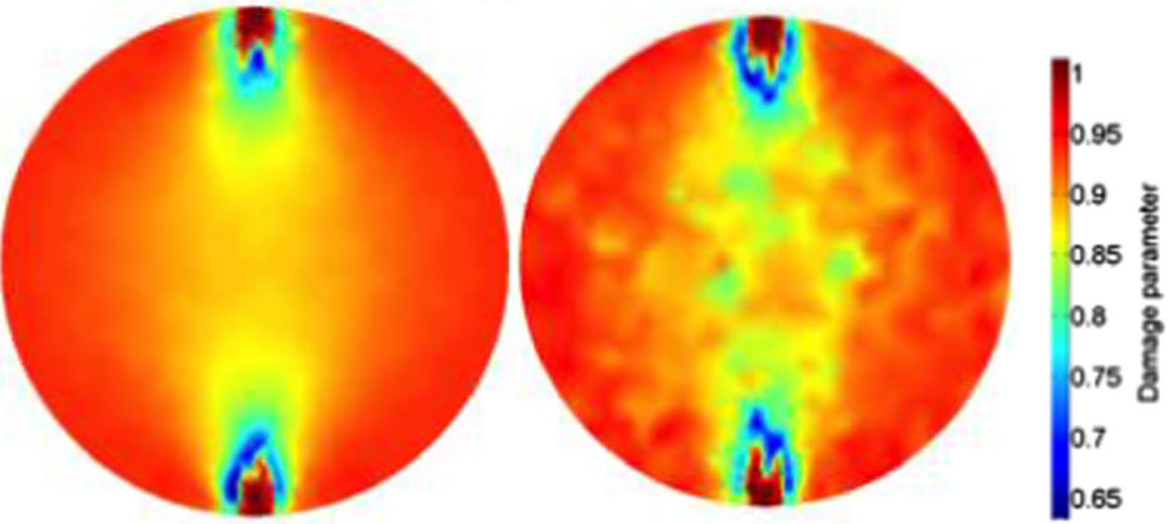

(c)
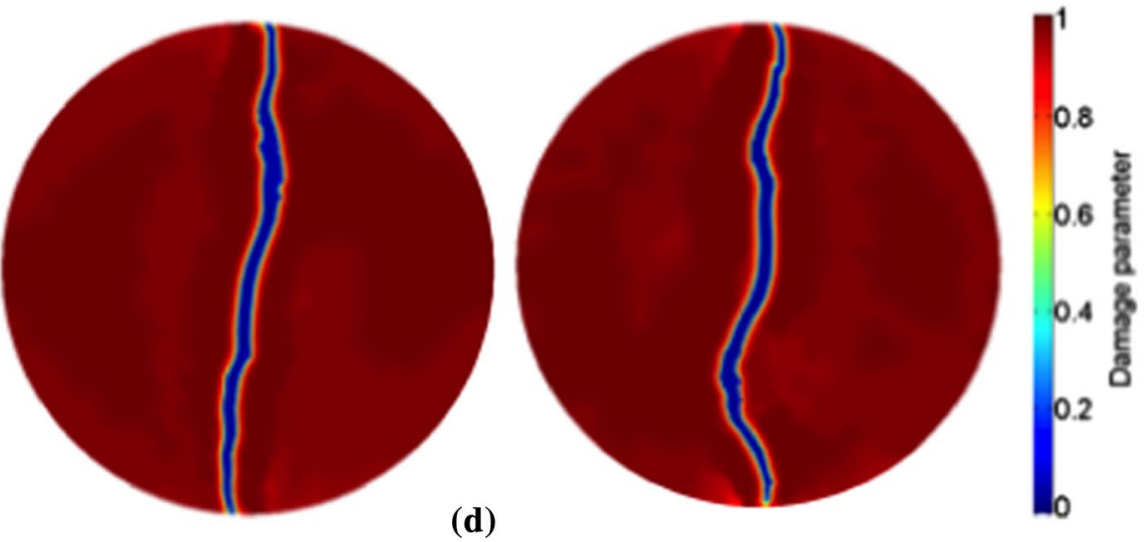


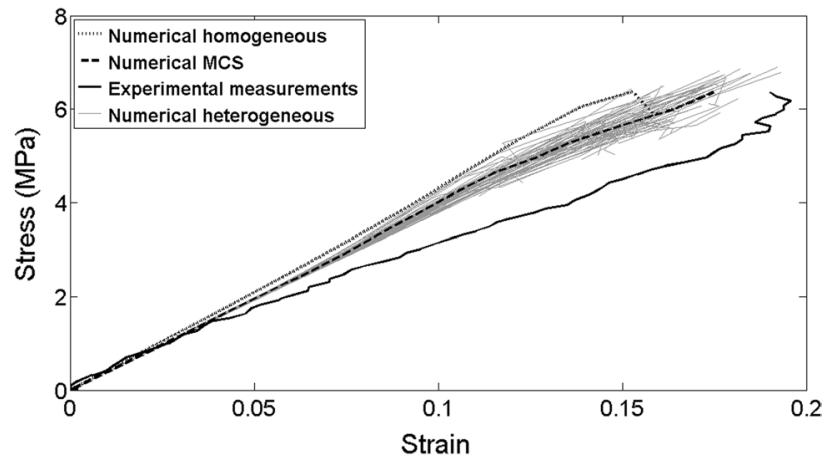

Fig. 25 Stress-strain plots for 100 simulations, their mean stressstrain curve and comparison between experimental data and numerical results from homogeneous and heterogeneous models (10\% standard deviation)

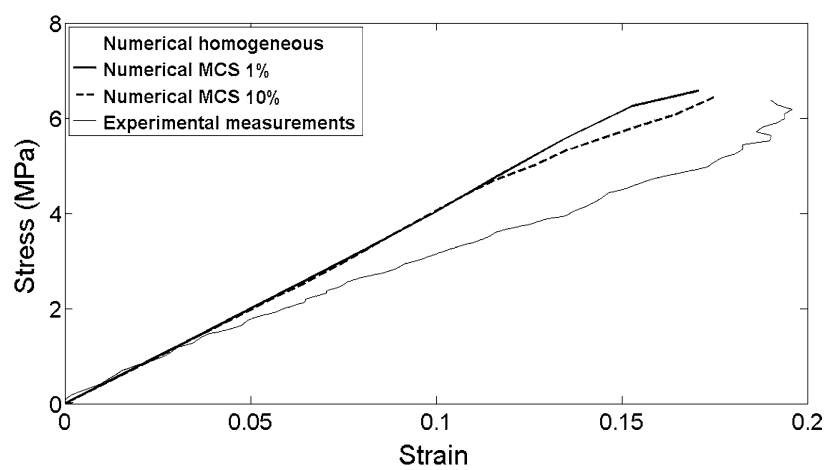

Fig. 26 Stress-strain curves and comparison between experimental data and numerical results from homogeneous and heterogeneous models with different degrees of standard deviation

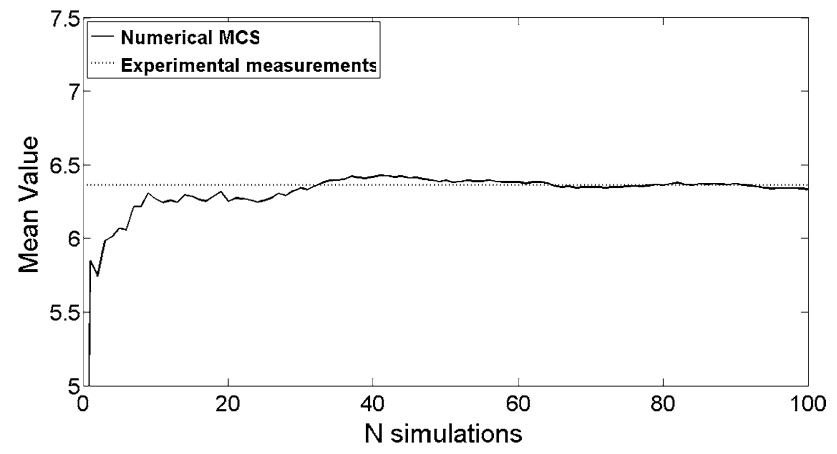

Fig. 27 Convergence of calculated mean value of tensile strength from simulations and comparison with the experimentally obtained tensile strength (10\% standard deviation)

\section{Conclusion}

Mechanical characterization of geomaterials such as shale rocks is still an open challenge, especially as their real behavior is affected by intrinsic heterogeneities and natural laminations. In this study, experimental data obtained from Brazilian tests conducted on intact shale-gas rock specimens were presented and used for the evaluation of material fracture energy, which was calculated on the basis of linear elastic fracture mechanics principles. Experiments on five different specimens were carried out in order to determine the values of rock samples' ultimate strength, elastic modulus and fracture toughness. Furthermore, the effects of bedding direction on crack paths and material resistance were also studied.

With regard to fracture toughness calculation, although the maximum tensile stress was found to be in the center of the specimen, in our experiments with flat plates the crack was initiating in the vicinity of the loading point. That was found to be the location of local maximum tensile strain, which combined with the effect of local heterogeneities, made the crack to initiate at the top of the shale sample. The calculated value of the fracture energy for that experiment with a low value of residual post-failure load was then used for the simulation of experimental tests. A new computational framework for modeling of crack propagation has been developed and numerically implemented. The numerical framework developed in this study considers the total energy of the body as the sum of strain energy and surface energy, and it is capable of capturing both crack initiation and crack propagation in materials. The comparison of results showed a very good agreement between the experimental observed and the numerical calculated crack paths. The wider difference between patterns was found to be in the proximity of the loading points, as this is the zone which is more affected by stress concentrations.

To include the rock heterogeneity in numerical simulations, three different strategies have been used: (1) different values for mechanical properties have been assigned to each layer of the numerical model, (2) mechanical properties have been defined as random fields, and (3) mechanical properties have been defined as random fields with a correlation length equal to the thickness of the layers of the numerical model. For the verification analysis different values of elastic modulus, Poisson's ratio and fracture energy were assigned to each layer, and different bedding directions were considered. Damage was localized between the interface of 

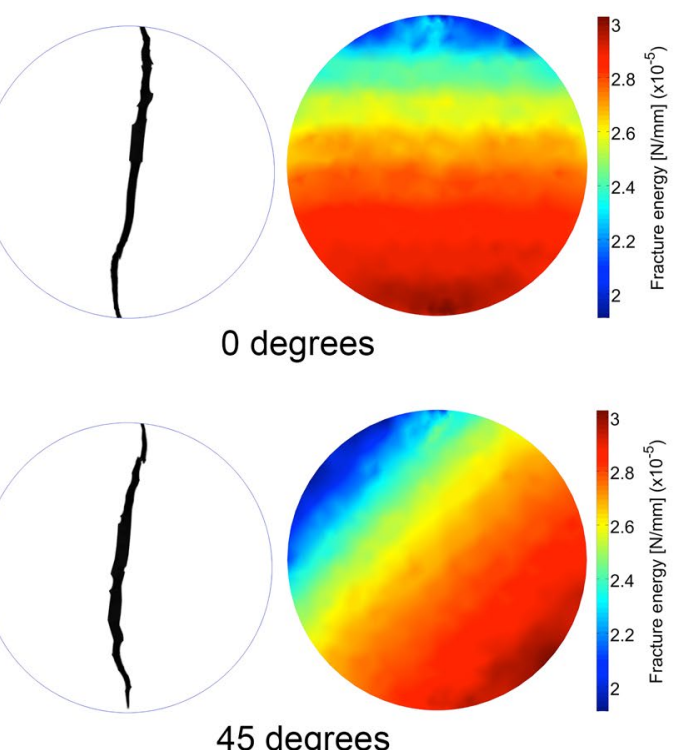

45 degrees

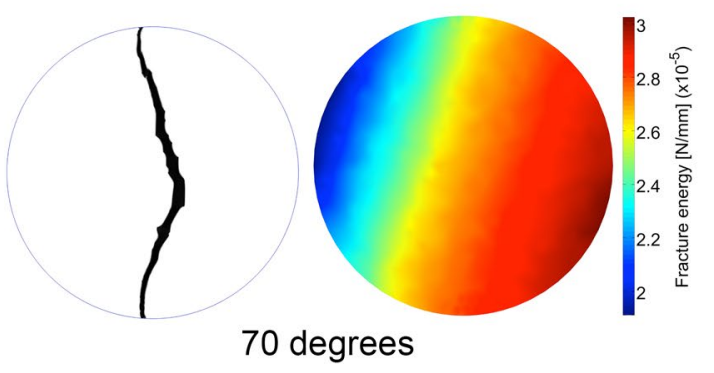

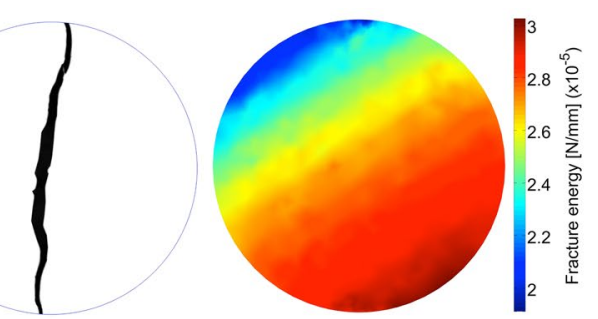

30 degrees

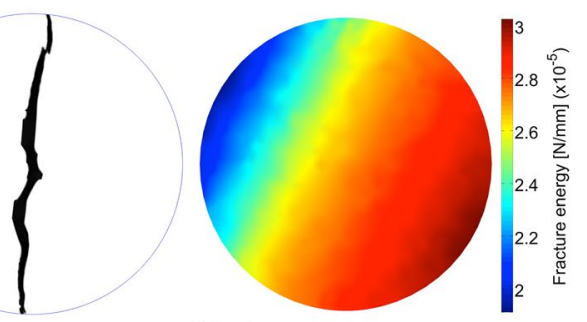

60 degrees

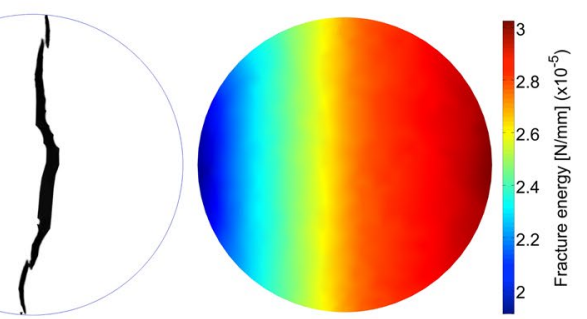

90 degrees

Fig. 28 Numerical crack patterns obtained with simulations of layered materials with different inclinations
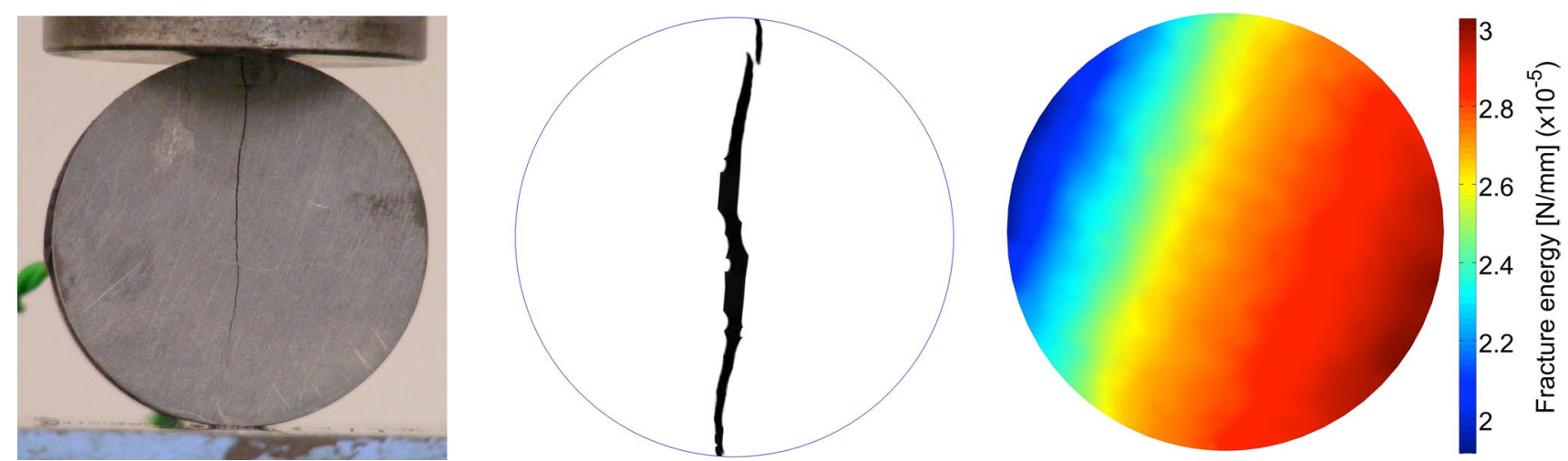

Fig. 29 Numerical crack path obtained with simulation of layered material $\left(67^{\circ}\right)$ and comparison with the experimental crack pattern

different layers, and crack patterns have shown tendency to move through the layers with lower toughness. The maximum value of resistance was obtained for an inclination of $0^{\circ}$, while the minimum was obtained for $30^{\circ}$ inclination. If different values for fracture energy were assigned to each element node, crack was observed to move toward weaker zones of the material.
It is worth noticing that inclusion of heterogeneity makes the simulation of mechanical behavior closer to the experimental data. Final material strength for layered materials is significantly influenced by bedding direction, and higher values of tensile strength are obtained for material with horizontal and vertical layers. If the layered nature of the material is incorporated by means of a combined approach which 


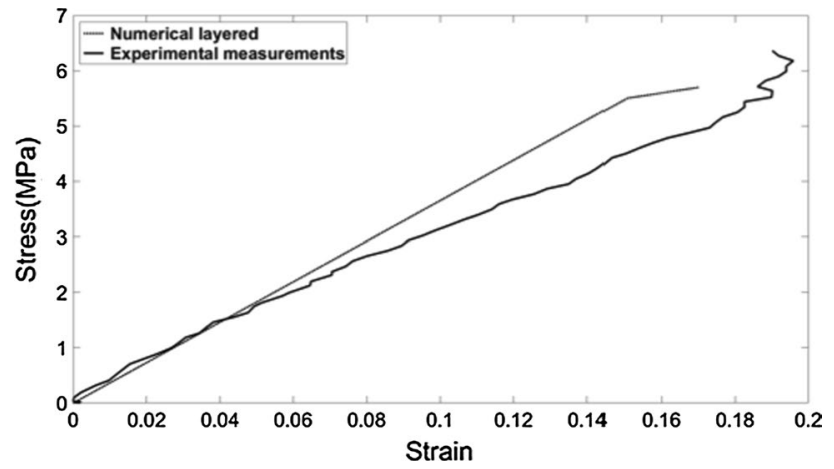

Fig. 30 Stress-strain cures of experimental data and numerical simulation with layers inclined of $67^{\circ}$

is considering both bedding direction and spatial variability, the crack path obtained could be closest to the experimental results.

Open Access This article is distributed under the terms of the Creative Commons Attribution 4.0 International License (http://creativeco mmons.org/licenses/by/4.0/), which permits unrestricted use, distribution, and reproduction in any medium, provided you give appropriate credit to the original author(s) and the source, provide a link to the Creative Commons license, and indicate if changes were made.

\section{References}

Aliha MRM, Ayatollahi MR, Smith D, Pavier MJ (2010) Geometry and size effects on fracture trajectory in a limestone rock under mixed mode loading. Eng Fract Mech 77(11):2200-2212

Ambrosio L, Tortorelli VM (1992) On the approximation of free discontinuity problems. Boll Un Mat Ital 6-B:105-123

Belytschko T, Loehnert S, Song JH (2008) Multiscale aggregating discontinuities: a method for circumventing loss of material stability. Int J Numer Methods Eng 73(6):869-894

Bobko C, Ulm FJ (2008) The nano-mechanical morphology of shale. Mech Mater 40(4):318-337

Borghi A, Renard P, Fournier L, Negro F (2015) Stochastic fracture generation accounting for the stratification orientation in a folded environment based on an implicit geological model. Eng Geol 187:135-142

Bossi G, Borgatti L, Gottardi G, Marcato G (2016) The Boolean Stochastic Generation method-BoSG: a tool for the analysis of the error associated with the simplification of the stratigraphy in geotechnical models. Eng Geol 203:99-106

Bourdin B, Francfort GA, Marigo JJ (2000) Numerical experiments in revisited brittle fracture. J Mech Phys Solids 48(4):797-826

Chen Q, Mousavi Nezhad M, Fisher Q, Zhu HH (2016) Multi-scale approach for modeling the transversely isotropic elastic properties of shale considering multi-inclusions and interfacial transition zone. Int J Rock Mech Min 30:95-104

De Giorgi E, Ambrosio L (1988) Un nuovo tipo di funzionale del calcolo delle variazioni. Atti Accad Naz Lincei Rend Cl Sci Fis Mat Nat 8(82):199-210

Del Piero G, Lancioni G, March R (2007) A variational model for fracture mechanics: numerical experiments. J Mech Phys Solids 55(12):2513-2537
Ding W, Li C, Li C, Xu C, Jiu K, Zeng W, Wu L (2012) Fracture development in shale and its relationship to gas accumulation. Geosci Front 3(1):97-105

Dokhani V, Yu M, Bloys B (2016) A wellbore stability model for shale formations: accounting for strength anisotropy and fluid induced instability. J Nat Gas Sci Eng 32:174-184

Duan K, Kwok CY (2015) Discrete element modeling of anisotropic rock under Brazilian test conditions. Int J Rock Mech Min 78:46-56

Ewy RT (2015) Shale/claystone response to air and liquid exposure, and implications for handling, sampling and testing. Int J Rock Mech Min Sci 80:388-401

Fang Z, Harrison JP (2002) Development of a local degradation approach to the modeling of brittle fracture in heterogeneous rocks. Int J Rock Mech Min 39(4):443-457

Geers MG, Kouznetsova VG, Brekelmans WAM (2010) Multi-scale computational homogenization: trends and challenges. J Comput Appl Math 234(7):2175-2182

Gironacci E, Mousavi Nezhad M, Rezania M, Lancioni G (2017) A nonlocal probabilistic method for modelling of crack propagation. Int J Mech Sci. https://doi.org/10.1016/j.ijmecsci.2017.11.015

Gorjan L, Ambrožič M (2012) Bend strength of alumina ceramics: a comparison of Weibull statistics with other statistics based on very large experimental data set. J Eur Ceram Soc 32(6):1221-1227

Guo H, Aziz NI, Schmidt LC (1993) Rock fracture-toughness determination by the Brazilian test. Eng Geol 33(3):177-188

Guy N, Seyedi DM, Hild F (2012) A probabilistic nonlocal model for crack initiation and propagation in heterogeneous brittle materials. Int J Numer Methods Eng 90(8):1053-1072

Hobbs DW (1964) The tensile strength of rocks. Int J Rock Mech Min 1(3):385IN17389-388IN18396

Holt RM, Fjær E, Stenebråten JF, Nes OM (2015) Brittleness of shales: relevance to borehole collapse and hydraulic fracturing. J Petrol Sci Eng 131:200-209

Hou P, Gao F, Ju Y, Liang X, Zhang Z, Cheng H, Gao Y (2016) Experimental investigation on the failure and acoustic emission characteristics of shale, sandstone and coal under gas fracturing. J Nat Gas Sci Eng 35:211-223

Hudson JA, Brown ET, Rummel F (1972) The controlled failure of rock discs and rings loaded in diametral compression. Int J Rock Mech Min 9(2):241IN1245-244IN4248

Kim H, Cho JW, Song I, Min KB (2012) Anisotropy of elastic moduli, $\mathrm{P}$-wave velocities, and thermal conductivities of Asan Gneiss, Boryeong Shale, and Yeoncheon Schist in Korea. Eng Geol 147:68-77

Knott JF, Elliott D (1979) Worked examples in fracture mechanics. Brookfield Pub Co. ISBN10: 9998970482

Li D, Wong LNY (2013) The Brazilian disc test for rock mechanics applications: review and new insights. Rock Mech Rock Eng 46(2):269-287

Li T, Marigo JJ, Guilbaud D, Potapov S (2016a) Gradient damage modeling of brittle fracture in an explicit dynamics context. Int $\mathrm{J}$ Numer Methods Eng 108(11):1381-1405

Li Z, Wang X, Wang H, Liang RY (2016b) Quantifying stratigraphic uncertainties by stochastic simulation techniques based on Markov random field. Eng Geol 201:106-122

Mahanta B, Tripathy A, Vishal V, Singh TN, Ranjith PG (2017) Effects of strain rate on fracture toughness and energy release rate of gas shales. Eng Geol 23:39-49

Mokhtari M, Tutuncu AN (2016) Impact of laminations and natural fractures on rock failure in Brazilian experiments: a case study on Green River and Niobrara formations. J Nat Gas Sci Eng 36:79-86

Morgan SP, Einstein HH (2014) The effect of bedding plane orientation on crack propagation and coalescence in shale. In: 48th US rock mechanics/geomechanics symposium. American Rock Mechanics Association, Minneapolis, Minnesota 1-4 June 2014 
Morgan SP, Johnson CA, Einstein HH (2013) Cracking processes in Barre granite: fracture process zones and crack coalescence. Int J Fract 180(2):177-204

Mousavi Nezhad M, Zhu H, Woody J, Chen Q (2016) A simplified multiscale damage model for the transversely isotropic shale rocks under tensile loading. Int J Damage Mech 25(5):705-726

Mousavi Nezhad M, Gironacci E, Rezania M, Khalili N (2018) Stochastic modelling of crack propagation in materials with random properties using isometric mapping for dimensionality reduction of nonlinear data sets. Int J Numer Methods Eng 113(4):656-680

Novák J, Kaczmarczyk Ł, Grassl P, Zeman J, Pearce CJ (2012) A micromechanics-enhanced finite element formulation for modeling heterogeneous materials. Comput Method Appl M 201:53-64

Stefanou G (2009) The stochastic finite element method: past, present and future. Comput Methods Appl M 198(9):1031-1051

Sun K, Zhang S, Xin L (2016) Impacts of bedding directions of shale gas reservoirs on hydraulically induced crack propagation. Nat Gas Ind B 3(2):139-145

Veytskin YB, Tammina VK, Bobko CP, Hartley PG, Clennell MB, Dewhurst DN, Dagastine RR (2017) Micromechanical characterization of shales through nanoindentation and energy dispersive X-ray spectrometry. Geomech Energy Environ 9:21-35

Wang J, Xie L, Xie H, Ren L, He B, Li C, Yang Z, Gao C (2016) Effect of layer orientation on acoustic emission characteristics of anisotropic shale in Brazilian tests. J Nat Gas Sci Eng 36:1120-1129
Warpinski NR, Smith MB (1989) Rock mechanics and fracture geometry. Recent Adv Hydraul Fract 12:57-80

Weibull W (1939) The phenomenon of rupture in solids. Generalstabens litografiska anstalts förlag, Stockholm

Wong LNY, Einstein HH (2009) Systematic evaluation of cracking behaviour in specimens containing single flaws under uniaxial compression. Int J Rock Mech Min 46(2):239-249

Yang Z, Xu XF (2008) A heterogeneous cohesive model for quasibrittle materials considering spatially varying random fracture properties. Comput Method Appl M 197(45):4027-4039

Zeng X, Wei Y (2017) Crack deflection in brittle media with heterogeneous interfaces and its application in shale fracking. J Mech Phys Solids 101:235-249

Zhong J, Shengxin LI, Yinsheng MA, Chengming YI, Chenglin LI, Zongxing LI, Xuan LI, Yong LI (2015) Macro-fracture mode and micro-fracture mechanism of shale. Pet Explor Dev 42(2):269-276

Zhou J, Zhang L, Pan Z, Han Z (2016) Numerical investigation of fluiddriven near-borehole fracture propagation in laminated reservoir rock using PFC $^{2 D}$. J Nat Gas Sci Eng 36:719-733 